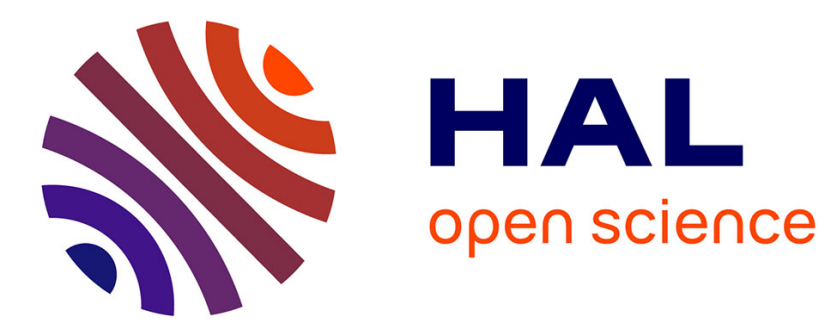

\title{
Stretching and mixing in sheared particulate suspensions
}

M. Souzy, H. Lhuissier, E. Villermaux, Bloen Metzger

\section{To cite this version:}

M. Souzy, H. Lhuissier, E. Villermaux, Bloen Metzger. Stretching and mixing in sheared particulate suspensions. Journal of Fluid Mechanics, 2017, 812, pp.611-635. 10.1017/jfm.2016.828 . hal01768671

\section{HAL Id: hal-01768671 \\ https://hal.science/hal-01768671}

Submitted on 19 Apr 2018

HAL is a multi-disciplinary open access archive for the deposit and dissemination of scientific research documents, whether they are published or not. The documents may come from teaching and research institutions in France or abroad, or from public or private research centers.
L'archive ouverte pluridisciplinaire HAL, est destinée au dépôt et à la diffusion de documents scientifiques de niveau recherche, publiés ou non, émanant des établissements d'enseignement et de recherche français ou étrangers, des laboratoires publics ou privés. 


\title{
Stretching and mixing in sheared particulate suspensions
}

\author{
M. Souzy ${ }^{1}$, H. Lhuissier ${ }^{1}$, E. Villermaux ${ }^{2, \dagger}$ \& B. Metzger ${ }^{1}$ \\ ${ }^{1}$ Aix Marseille Université, CNRS, IUSTI UMR 7343, 13453 Marseille, France \\ ${ }^{2}$ Aix Marseille Université, CNRS, Centrale Marseille, IRPHE UMR 7342, 13384 Marseille, \\ France \\ ${ }^{\dagger}$ Institut Universitaire de France, Paris
}

(Received 23 November 2016)

We experimentally investigate mixing in sheared particulate suspensions by measuring a crucial kinematic quantity of the flow: the stretching laws of material lines in the suspending liquid. High-resolution PIV measurements in the fluid phase are performed to reconstruct, following the Diffusive Strip Method (Meunier 2010), the stretching histories of the fluid material lines. In a broad range of volume fractions $20 \% \leqslant \phi \leqslant 55 \%$, the nature of the elongation law changes drastically from linear, in absence of particles, to exponential in the presence of particles: the mean and the standard-deviation of the material line elongations are found to grow exponentially in time and the distribution of elongations converges to a log-normal. A multiplicative stretching model, based on the distribution of local shear-rates and on their persistence time, is derived. This model quantitatively captures the experimental stretching laws. The presence of particles is shown to accelerate mixing at large Péclet numbers $\left(\gtrsim 10^{5}\right)$. However, the wide distribution of stretching rates results in heterogeneous mixing and hence, broadly distributed mixing times, in qualitative agreement with experimental observations.

\section{Introduction}

Sheared particulate suspensions represent a quasi-unique system where efficient dispersion spontaneously occurs even under low Reynolds number flow conditions. For instance, the transfer of heat (Sohn 1984, Metzger 2013) or mass (Wang et al. 2009, Wang \& Keller 1985, Souzy 2015) across a suspension of non-Brownian particles is significantly enhanced when the suspension is submitted to a macroscopic shear. This would not happen in a pure Newtonian fluid where the laminar streamlines remain perpendicular to the scalar (heat or concentration) gradients. In a sheared suspension, the macroscopic stationary imposed shear results at the particle scale in an unstationary flow: particles constantly collide with one another, change streamlines and thus generate disturbances within the fluid which promote the dispersion of the scalar, prelude to its subsequent mixing. Two mechanisms have been identified to explain the origin of the transfer enhancement. First, the particle translational shear-induced diffusivity, a phenomenon which has been widely investigated over the last decades (Eckstein 1977, Arp 1976, Da Cunha 1996, Breedveld 2002, Sierou 2004, Metzger 2013). Second, the particle rotation whose impact is particularly important at the boundaries, where particles disrupt the diffusive boundary layer by a 'rolling-coating' effect (Souzy 2015). These studies mainly focused on the rate of transfer across sheared suspensions which is customary characterized by an effective diffusion coefficient much larger than the scalar molecular diffusivity. 
a)

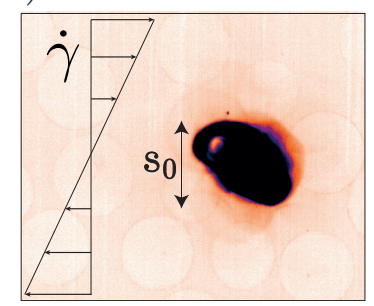

b)

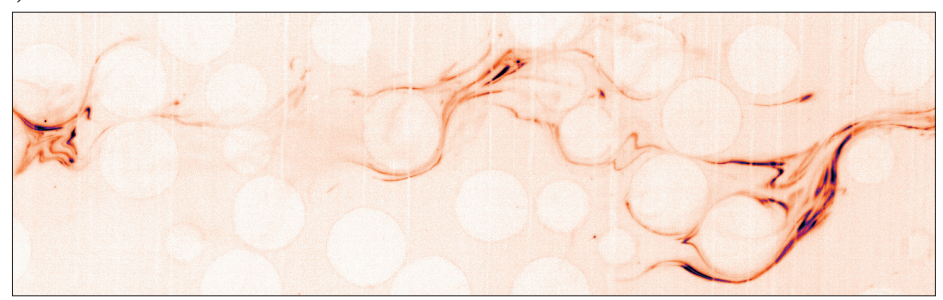

Figure 1. Some dye, initially confined to a small blob in a flowing particulate suspension (a), mixes with the rest of the suspension (b) by diffusing while the blob is stretched in the complex micro-flow generated by the particles.

Another aspect of transport enhancement concerns the mixing properties of the system, namely its ability, starting from a given spatial scalar distribution, to reach homogeneity. Figure 1 shows how a blob of dye with initial size $s_{0}$, diffuses while it is deformed by the complex flow in the interstitial fluid of a suspension. The important question which naturally arises is to understand how this initially segregated system reaches homogeneity, and particularly how long this process takes. By essence, it involves both advection by the flow and molecular diffusion of the scalar. Such a problem has been studied in a wide range of situations involving a single fluid phase such as shear flows (Ranz 1979), vortice flows (Meunier 2003), turbulent jets (Duplat 2010), or flows in porous media (Le Borgne 2015). These studies all underline the crucial importance of the rate at which fluid material lines are elongated by the flow (Villermaux 2012). The knowledge of these 'stretching laws' allows to estimate the mixing time: the time when the scalar concentration fluctuations start to significantly decay (Batchelor 1959). For instance, in a simple shear flow with rate $\dot{\gamma}$, the material lines grow as $\dot{\gamma} t$. In the limit of large Péclet number $P e=\dot{\gamma} s_{0}^{2} / D$, the mixing time for a scalar blob of initial size $s_{0}$ is $t_{\text {mix }} \sim \dot{\gamma}^{-1} P e^{1 / 3}$, where $D$ denotes the molecular diffusivity of the dye. In chaotic flows, where the stretching rate is maintained, the material lines stretch exponentially, as $\mathrm{e}^{\dot{\gamma} t}$, and $t_{\mathrm{mix}} \sim \dot{\gamma}^{-1} \ln P e$.

In spite of their crucial importance for mixing issues, stretching laws in particulate suspensions have never been studied experimentally, nor have the general question about the mixing time in such a system been. Stretching in particulate suspension has been addressed indirectly using numerical simulations through the measurement of the suspension largest Lyapunov exponent (Dasan 2002, Drazer 2002, Metzger 2012, Metzger 2013). In such a chaotic system, the mean stretching rate of fluid elements can be assimilated to the largest Lyapunov exponent. The reported positive Lyapunov exponents indicate that the stretching laws must be exponential. Stretching has also been explored theoretically with the motivation of understanding the rheology of such system when the suspending fluid is viscoelastic. It was shown that the expected exponential stretching of the polymers should affect the pressure drop in fixed beds of spheres or fibres (Shaqfeh 1992) or the viscosity of freely suspended fibres in a simple shear flow (Harlen 1993).

In this paper, we specifically address the question of the stretching kinematics by performing experiments on non-Brownian and spherical particulates suspended in a viscous and Newtonian fluid that is steadily and uniformly sheared. In this limit, the flow kinematics is independent of both the shear rate $\dot{\gamma}$ and the molecular diffusivity. The sole parameter expected to affect the stretching process is the particulate volume fraction $\phi$. We investigate the stretching laws in particulate suspensions varying the volume fraction over a wide range $20 \% \leqslant \phi \leqslant 55 \%$, for which collective effects between particles are present but the suspension still flows easily, since it is still far from jamming. After 

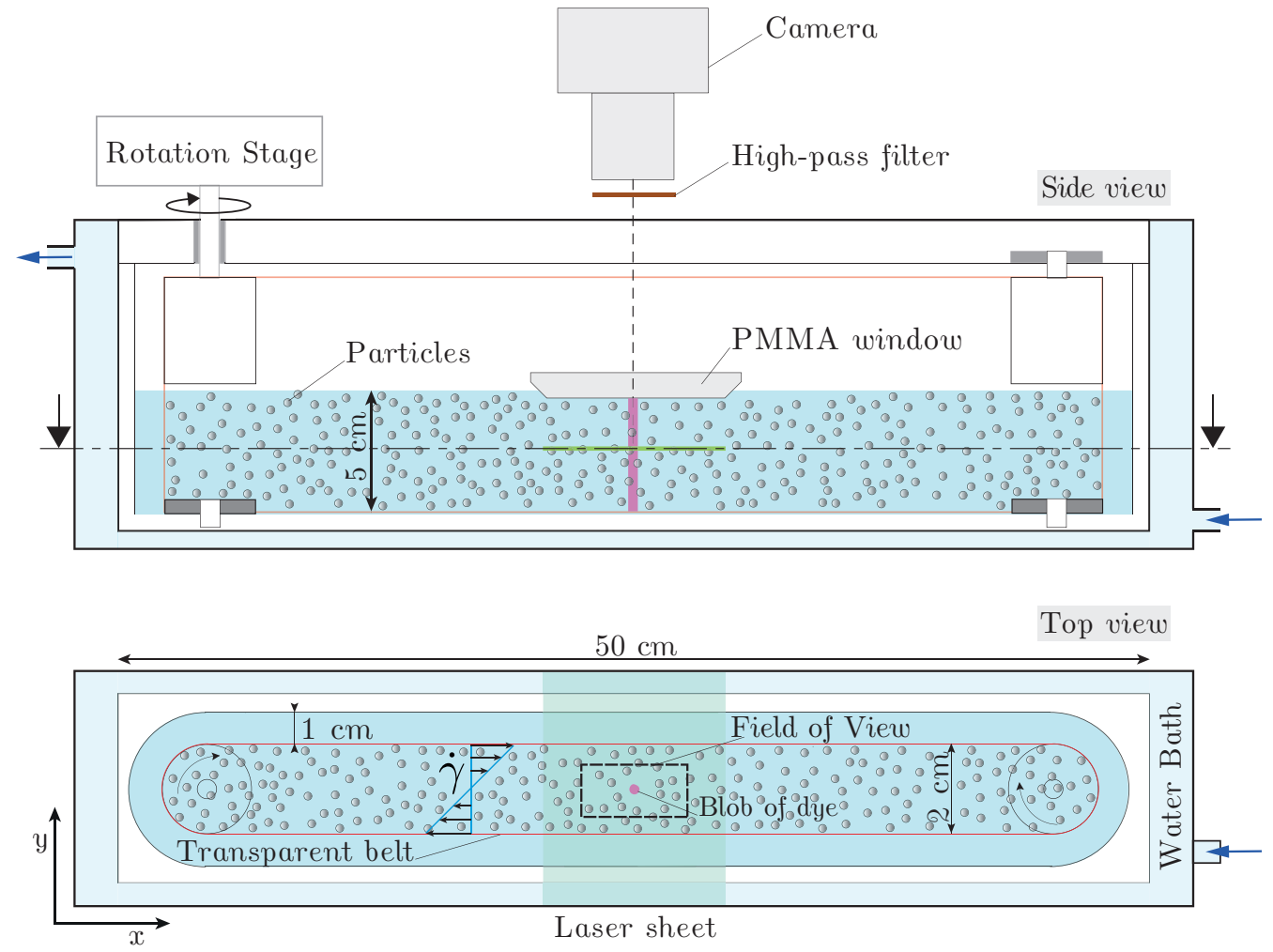

FiguRE 2. Schematics of the set-up.

presenting the experimental set-up in $\S 2$, we first compare the evolution of a blob of dye sheared in a pure fluid (without particles) to that of a blob sheared in a suspension (§ 3). This experiment illustrates the complexity of the advection field induced by the presence of the particles. Then, following the Diffusive Strip Method of Meunier 2010, accurate velocity field measurements of the fluid phase $(\S 2.3)$ are used to determine the stretching laws. Material lines are found to stretch, on average, exponentially with time $(\S 4)$, at a rate which agrees with the largest Lyapunov exponents reported in 3D Stokesian dynamic simulations (Drazer 2002, Dasan 2002). Beyond the mean, we tackle the complete statistics of stretching, that is to say, the distributions of elongation as a function of strain and particle volume fraction, which are found to converge towards log-normal distributions. In $\S 5$, we present a model, based on a multiplicative stretching process, which explains quantitatively the experimental distributions of the material line elongation and its dependance to $\dot{\gamma} t$ and $\phi$. Finally, the crucial implication of these findings for scalar mixing are developed and discussed in $\S 6$, before we conclude in $\S 7$.

\section{Experimental set-up}

The experimental set-up is shown in figure 2. It aims at steadily and uniformly shearing a viscous particulate suspension, injecting a small blob of dyed fluid, and observing both the flow and the mixing of the dye. The set-up consists of a transparent cell in which a transparent mylar belt is tightly mounted at the top of the cell on two cylinders and at the bottom on two ball bearings. One cylinder is entrained by a rotating stage (M-061.PD from PI Piezo-Nano Positioning) with high angular resolution $\left(3 \times 10^{-5} \mathrm{rad}\right)$. The motion 
of the belt generates in its central region a linear shear flow. The suspension is allowed to flow below the cylinders and a constant spacing between the belt and the inner wall of the cell is maintained all around the cell. This specific design, which is an evolution of that used in Metzger 2012, minimizes secondary flows and ensures a velocity profile with constant shear rate within the belt.

\subsection{Particles and liquid}

The particles and the liquid are carefully chosen to allow the visualization of the dye and of the flow inside the suspension, as well as to ensure a purely viscous flow without buoyancy effects. This requires using a transparent media, matching both the density and the refraction index of the particles, and using a fairly viscous liquid.

To fulfill the above requirements, we use mono-disperse spherical particles (PMMA from Engineering Laboratories Inc.) with density $\rho=1.18 \mathrm{~kg} / \mathrm{m}^{3}$ and diameter $d=2$ $\mathrm{mm}$, especially chosen for their smooth surface and good transparency. The liquid is a Newtonian mixture of Triton X-100 (77.4 wt \%), Zinc Chloride (13.4 wt \%) and water $(9.2$ wt \%) with viscosity $\eta=3 \mathrm{~Pa} \mathrm{~s}$ and having the same density as the particles at room temperature. Its composition is optimized to match both the refractive index and the density of the particles. A small amount of hydrochloric acid $(\approx 0.05 \mathrm{wt} \%)$ is added to the solution to prevent the formation of zinc hypochlorite precipitate, thereby significantly improving the optical transparency of the solution. Last, to finely tune the index matching between the particles and the liquid, the temperature of the set-up is adjusted with a water bath surrounding the shear cell.

The solid volume fraction $\phi$ of the suspension is varied between 20 and $55 \%$. To ensure that inertial effects are negligible, the shear rate $\dot{\gamma}$ is set to typically $0.15 \mathrm{~s}^{-1}$, which corresponds to a particulate Reynolds number $\rho \dot{\gamma} d^{2} / \eta \sim 10^{-4}$.

\subsection{Imaging}

The suspension is observed in the flow-gradient plane ( $x y$ plane): a slice of suspension is illuminated by a laser sheet across the transparent belt and imaged from the top (see figure 2).

The laser sheet is formed by reflecting a laser beam $(2 \mathrm{~W}, 532 \mathrm{~nm})$ on a standard laserprinter mirror (rotating at $\sim 10000 \mathrm{rpm}$ ). This technique was found to produce a light sheet with a better spatial homogeneity than that obtained with classical cylindrical or Powel lense techniques. The sheet is collimated and focused to a thickness of $\sim 60 \mu \mathrm{m}$ with the help of two perpendicular plano-convex lenses. Last, a high-pass filter $(590 \mathrm{~nm})$ eliminates direct light reflexions. The suspension is imaged with a high-resolution camera (Basler Ace2000-50gm, 2048x1080 pixel $^{2}$, 12bit) coupled to a high-quality magnification lens (Sigma APO-Macro-180 mm-F3.5-DG). To avoid that the particles distord the free surface of the suspension through which the visualization is realized, a small plexiglass window is positioned on the free surface, above the region of interest, which locally ensures a flat interface. The window has a small hole allowing the injection of a blob of dyed fluid with a syringe.

\subsection{Velocity field measurements}

The velocity field in the suspending liquid is measured in the plane of the laser sheet $(x y)$, at half-distance between the bottom and the free surface (see figure 3), performing particle image velocimetry (PIV), which yields the two-dimensional velocity field $\{u, v\}$, which does not necessarily verify incompressibility. To perform PIV, the liquid is seeded with small passive fluorescent tracers (3.23 $\mu \mathrm{m}$ PMMA B-particles from MF-Rhodamine) at a very low volume fraction $\left(\sim 10^{-5} \ll \phi\right)$. These small and diluted tracers do not affect 
a)

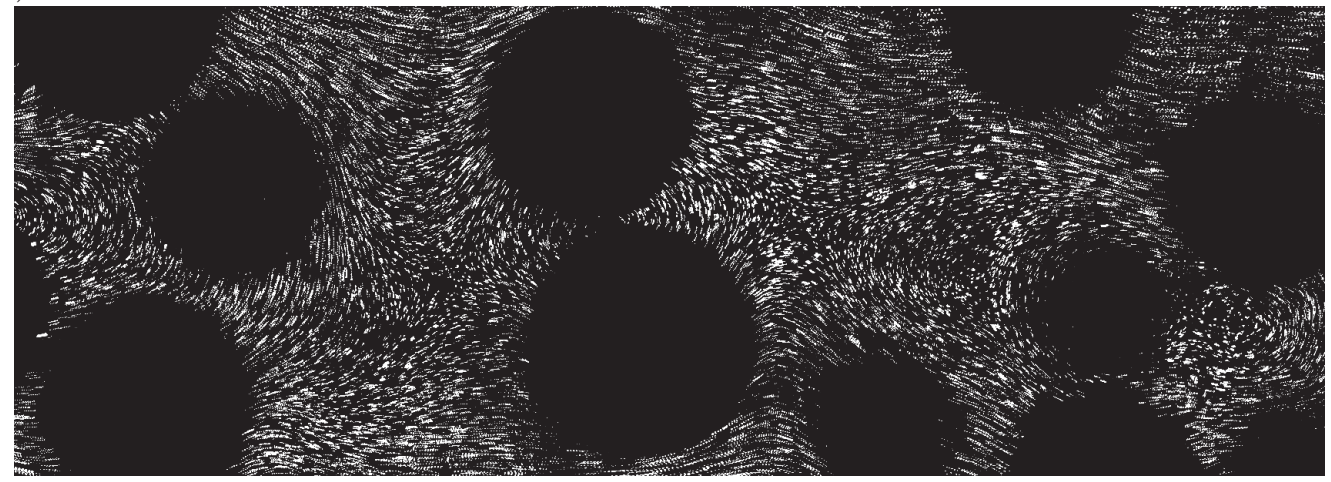

b)

c)

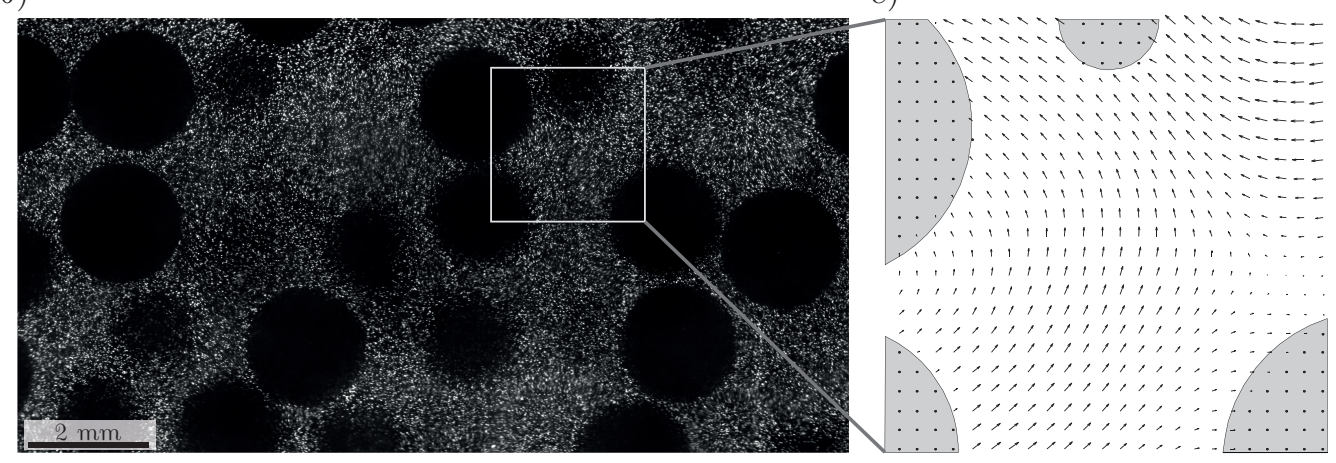

FIGURE 3. a) Flow streamlines in the bulk of a suspension. b) Slice of a sheared suspension illuminated by a laser sheet. The small fluorescent tracers seeding the suspending fluid appear as bright whereas the particle intersections with the laser sheet appear as dark, see also Movie 1. c) Magnified view of the suspending liquid velocity field obtained from the PIV (the velocity is not computed in the particles).

the flow but allow its visualization and quantification, as shown in figure 3 and Movie 1. The large $(2 \mathrm{~mm})$ particles of the suspension do not interact with the laser sheet and appear as black discs. Note that all the particles have the same size; the apparent size differences arise from their different vertical positions relative to the laser sheet plane. The PIV routine is adapted from a Matlab code developed by Meunier 2003. Images are captured every $0.1 \mathrm{~s}$, which corresponds to a strain increment of 0.015 . To perform PIV, the images are divided into equally spaced and overlapping sub-images with a typical size of $d / 20$ (32 pixels). The local velocity field is computed by cross-correlating successive sub-images. The presence of a particle in the sub-image is detected with the help of two filters (for the maximum of correlation and for the standard deviation of the sub-images), in which case the corresponding velocity vector is not used (see figure $3 \mathrm{~b})$. For each volume fraction, three independent runs over a strain of 20 are performed. The independence of the measured velocity field on the PIV sub-image size was verified by decreasing the latter to $\sim d / 40$ (16 pixels). Besides increasing the data noise, no significant effect was found on the measured velocities.

\subsection{Molecular diffusivity measurements}

The molecular diffusion coefficient of the dye (rhodamine $6 \mathrm{G}$ ) is measured by observing the spreading, in the absence of flow, of a slice of liquid depleted in dye. A small HeleShaw cell $(100 \mu \mathrm{m}$ thick $)$ is filled with dye-doped suspending liquid without particles. A 
a)

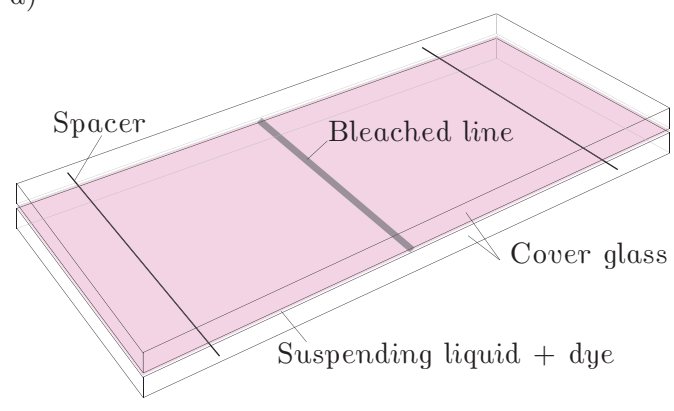

b)

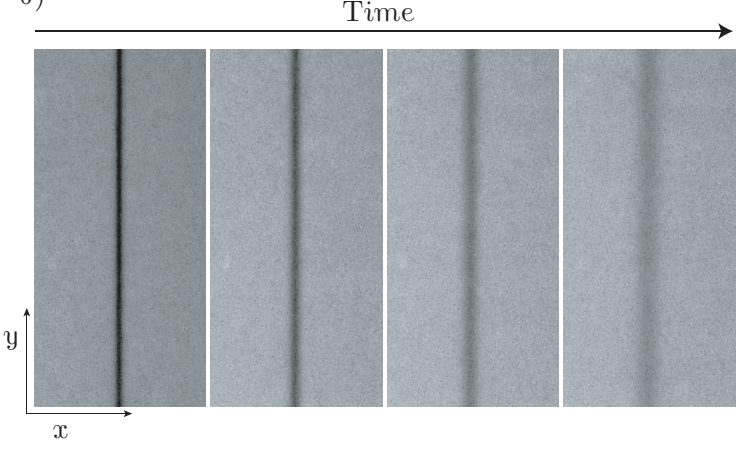

c)
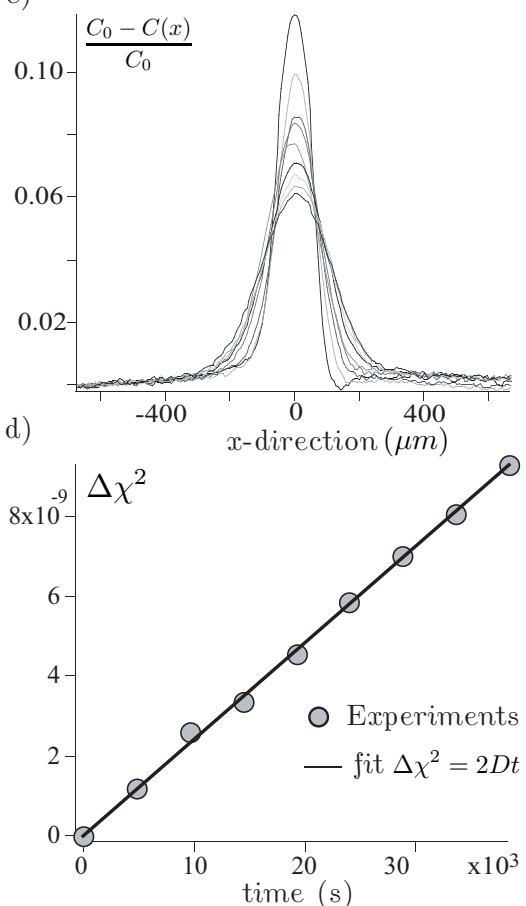

FiguRE 4. a) Schematics of the set-up used to measure the molecular diffusivity $D$ of the dye (rhodamine 6G), in the suspending liquid (TritonX $+\mathrm{ZnCl}_{2}+\mathrm{H}_{2} \mathrm{O}$ ). b) Diffusive thickening of the bleached line at $t=0,4800,21600$ and $64800 \mathrm{~s}$ (the image width is $5 \mathrm{~mm}$ ). c) Concentration profiles at successive times $(0 \mathrm{~s}<t<64800 \mathrm{~s})$. d) Increase of the spatial variance of the concentration $\chi^{2}(t)-\chi^{2}(0)$ versus time. Its fit to $2 D t$ yields $D=1.44 \pm 0.2 \times 10^{-13} \mathrm{~m}^{2} \mathrm{~s}^{-1}$.

thin slice of liquid is initially depleted in dye by bleaching the dye with a high power laser sheet across the cell (see figure 4). The depleted slice appears as a dark line having a gaussian profile which diffuses with the diffusion coefficient of the dye. The spatial variance of the gaussian profile $\chi^{2}$ is measured over one day, and the diffusivity is determined from $D=\left[\chi^{2}(t)-\chi^{2}(0)\right] / 2 t \simeq 1.4410^{-13} \mathrm{~m}^{2} \mathrm{~s}^{-1}$. This value is consistent with that of $4.1410^{-10} \mathrm{~m}^{2} \mathrm{~s}^{-1}$ found by Culbertson 2002 for the diffusivity of the same dye in water, given that water is 3000 times less viscous than the suspending liquid and that, according to the Einstein-Sutherland law, $D \propto 1 / \eta$.

\section{General observations}

To illustrate the influence of particles on mixing in a shear flow, we first compare the evolution of a blob of dye sheared in a pure fluid (without particles) to that of a blob sheared in the suspension of particles, see Movie 2. A cylindrical blob of dyed fluid is injected, at rest and at $t=0$, in the middle of the shear cell. Initially, the blob has a diameter $s_{0} \simeq 2 \mathrm{~mm}$, is aligned with the vorticity direction and is centered on the neutral velocity plane. This results in a macroscopically two-dimensional initial configuration, and ensures that the blob does not drift with the flow but only deforms. Figure 5 shows, for a Péclet number $P e \approx 10^{6}$, how mixing proceeds in the two sheared media, from the initial segregated state up to a strain $\dot{\gamma} t=20$. In the pure liquid, the blob of dye stretches homogeneously. Its length increases linearly with time and the blob transverse 
a)

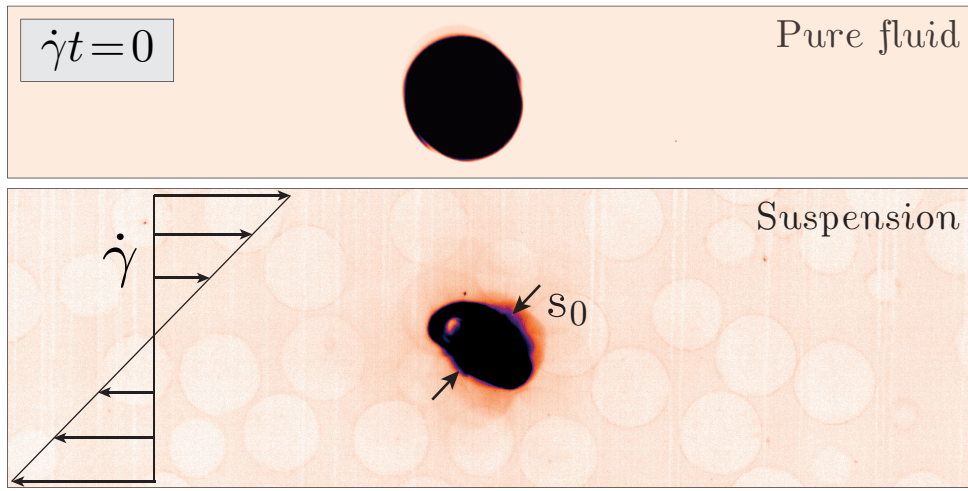

b)
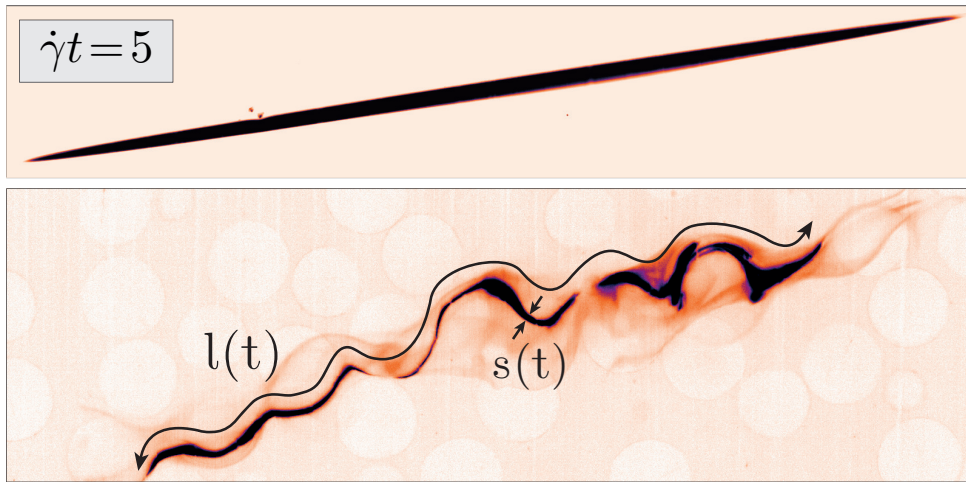

c)

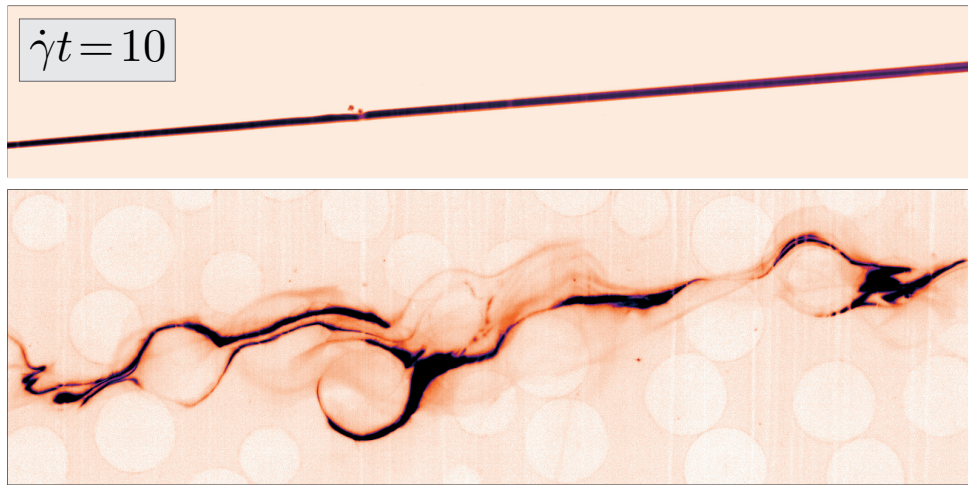

d)
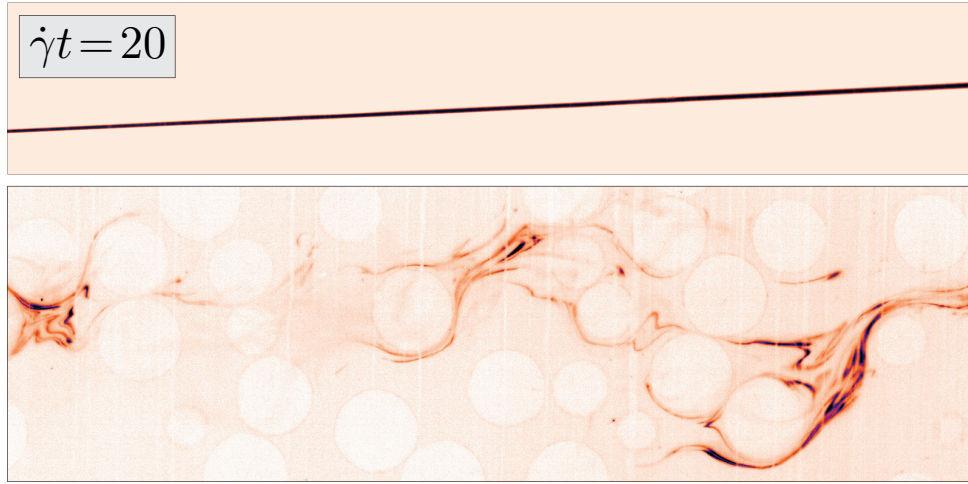

Figure 5. Comparison of the stretching processes of a blob of dye sheared at high Péclet $\left(\sim 10^{6}\right)$ and low Reynolds numbers $\left(\sim 10^{-4}\right)$, in a pure fluid (top), and in a particulate suspension with volume fraction $\phi=35 \%$ (bottom). The dye appears as dark, and the beads appear as bright, see also Movie 2. 
dimension thus decreases as $1 / t$. In the suspension, the situation is markedly different: the fluctuations induced by the particles in the fluid phase strongly impact the evolution of the blob. Several conspicuous features deserve being highlighted i) the dispersion and the unfolded length of the blob are significantly enhanced by the particles, ii) both translational diffusivity (transverse undulations of the blob, see figure 5b) and rotation (blob winding around particles, see figure 5c) of the particles contribute to these enhancements, iii) the blob stretching is highly inhomogeneous: at some locations, its transverse dimension becomes much thinner and at others larger than in the pure fluid case, revealing regions of enhanced stretching and regions of compression, iv) at large strains (figure 5d), the blob has separated into several filaments which means that some regions of the blob have already mixed, while in the pure liquid (without particles) mixing has not occurred yet, v) In some regions, the blob evolves into bundles composed of several nearly overlapping filaments (Duplat 2008). This suggests an underlying stretching/folding mechanism similar to the well known baker's transform (Ottino 1989).

The above features are generic to the flow of a viscous suspension at large Péclet number. Since inertial effects are negligible, these features are independent of the rate $\dot{\gamma}$ at which the suspension is sheared. Similarly, the value of the Péclet number does not influence the general stretching pattern of the blob, but only prescribes the strain $\dot{\gamma} t$ at which diffusion starts to becomes effective.

This direct comparison clearly illustrates how the liquid velocity fluctuations generated by the particles dramatically accelerate the blob deformation and dispersion. This acceleration is apparent here from the beginning of the shear, because the blob size $s_{0}$ is similar to the particle size $d$. It is however crucial to realize that the strain at which this acceleration establishes is expected to depend on the ratio $s_{0} / d$. If the initial size of the blob $s_{0}$ is larger than $d$, the blob is essentially not stretched by the particle fluctuation motions. It is thus essentially stretched by the linear macroscopic shear until the blob transverse size has thinned down to $d$, after a typical strain $s_{0} / d$. From that strain on, the particulate fluctuations are expected to contribute directly to the blob stretching.

In stirred flows, such as the case considered here, mixing results from the coupling between advection and molecular diffusion. In the experiment described above, the blob of dye is stretched by the local velocity field: the blob is stretched along its own longitudinal direction and conversely compressed along its transverse direction. The blob thus evolves towards a topology constituted of sheets, or filaments (Ottino 1989, Buch 1996). Conversely to the effect of advection, molecular diffusion tends to broaden the filaments. This diffusive broadening will at some point counter-balances the rate of compression of the blob caused by the advection. As we already mentioned, this naturally sets a time-scale called the mixing time, $t_{\text {mix }}$, beyond which the concentration levels drop significantly. The mixing time, a key element to understand the overall mixing process, can be estimated from the sole knowledge of the dye molecular diffusion coefficient and from the history of the transverse dimension of the blob. If one assumes that the flow is two-dimensional (this assumption is discussed in 5.3), incompressibility and mass conservation relate at any time the transverse size of the blob to its length $l$, through $s_{0} l_{0}=s(t) l(t)$. The mixing time can therefore be estimated from the characterization of the evolution of $l(t)$. Our goal in the following is thus to determine the so-called 'stretching laws', i.e., the time dependence of $l$ in sheared particulate suspensions.

\section{Experimental stretching laws}

Our first attempt to measure the unfolded length of the blob $l(t)$ was naturally to perform direct image analysis on images such as those shown in Figure 5. However, the 


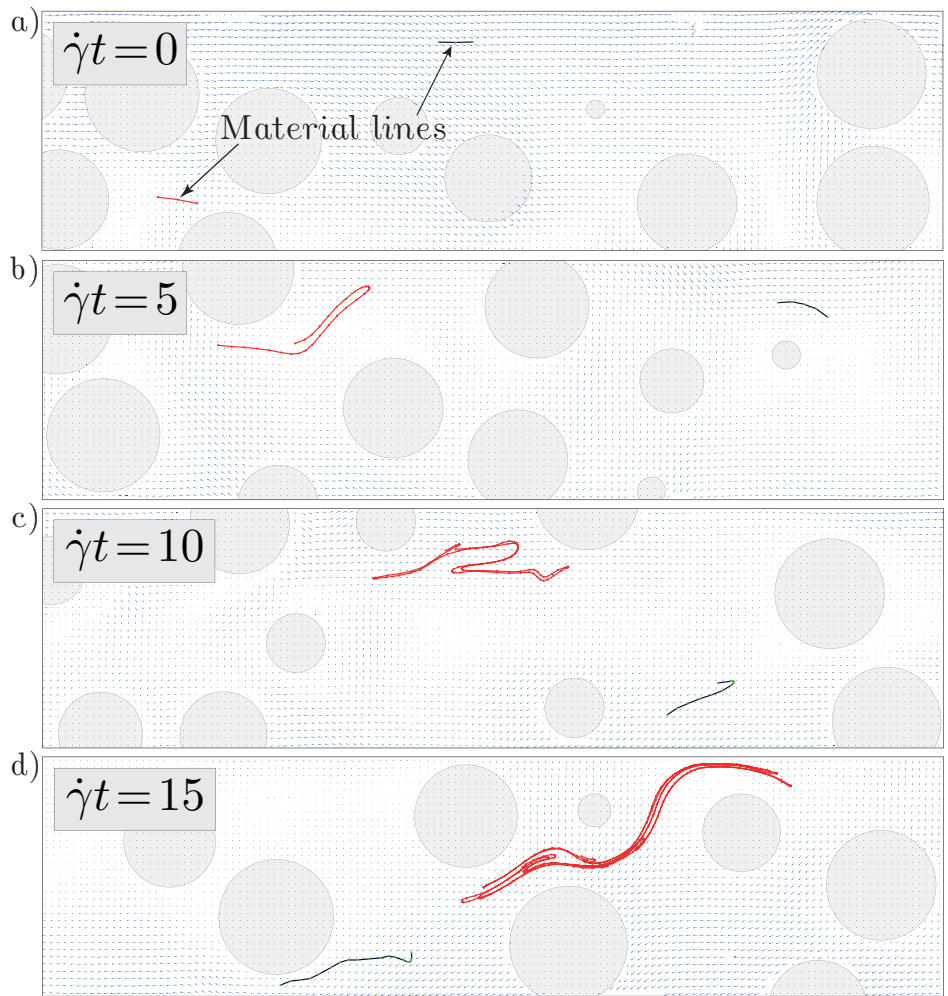

FiguRE 6. Example of stretching for two material lines numerically advected using the experimental fluid velocity field, see also Movie 3.

intrinsic dispersion process rapidly distorts the blob into bundles of very close (sometimes merging) filaments, which renders image analysis ineffective above strains of typically 5 .

To overcome these limitations, we adopted a different approach inspired from the Diffusive Strip Method. This method happens to be a very powerful experimental tool allowing the determination of the stretching laws over unlimited strains. The key idea is to use the experimental fluid velocity field to numerically advect passive material lines representing portions of the blob. The lines are initially composed of three passive tracers separated by a distance $d / 20$ which discretize a fluid material line. The lines are randomly located in the two-dimensional velocity field with a random orientation (see figure 6a). Each tracer with coordinate $\mathbf{x}$ is advected independently from each other according to the local fluid velocity $\mathbf{v}(\mathbf{x})$ (obtained by linear interpolation of the instantaneous PIV velocity field) as $\mathbf{x}(t+\Delta t)=\mathbf{x}(t)+\mathbf{v}(\mathbf{x}) \Delta t$, where $\Delta t$ is the time between consecutive measurements of the velocity field. As a material line is advected, it is refined by adding more tracers when its length increases or when its local curvature becomes too large (see Meunier 2010 for a detailed description of the refinement procedure).

Figure 6 shows the evolution of two material lines up to a strain of 15, see also Movie 3 . The red line successively stretches and folds very similarly to what is observed in the blob experiments (figure 5). Interestingly, the blue line behaves very differently. Although it sustains the same macroscopic strain as the red one, it experiences a much softer stretching only because it started from a different initial location. These different stretching histories reveal the stochastic nature of the stretching induced within particulate suspensions. The stretching laws therefore have to be sought in a statistical sense 
by repeating the advection procedure over a large number of independent material lines. However, as the material lines lengthen, they may reach the boundaries of the measured velocity field, which limits the maximum strain that can practically be investigated (typically $\dot{\gamma} t<10$ ). This problem is easily circumvented by realizing that, as long as the stretching laws are concerned, the object of interest is not the material line as a whole but rather the small segments which compose this line and which all stretch differently from each other. We thus perform a new set of calculations focusing on segments: i) initial segments (composed of two tracers) with length $d / 20$ are positioned and oriented randomly in the flow, ii) each time the length of a segment doubles, it is splited in two individual segments that are subsequently advected independently, iii) if a segment reaches the boundary of the velocity field, it is re-injected near the center of the velocity field, iv) when a segment overlaps with a particle, where the velocity field is undefined (as can happen due to the finite time $\Delta t$ ), it is frozen until the particle has moved away. Owing to these rules, virtually unlimited strains can be considered, and the stretching history of each segment that have been created over this strain can be determined. We define the elongation of these segments as the ratio

$$
\rho(t) \equiv \delta l(t) / \delta l_{0}
$$

of their current length $\delta l(t)$ to their initial length $\delta l_{0}$ where $\delta l_{0}=(d / 20) / 2^{n}$, with $n$ the number of times the sub-segment was splited in two. Note that to compute the distributions of elongations we present below, the contribution of each segment is weighted by its initial length. Note also that times for which a segment is frozen are not considered. The distribution of elongations at time $t$ therefore represents the portion of the blob that has reached a given elongation after being advected for a duration $t$. It was built from the stretching histories of 25000 segments advected over 3 independent experimental velocity fields, each of them recorded for a total strain of 20 (typically 4000 images).

Figure 7 shows the experimental stretching laws obtained for a suspension with $\phi=$ $35 \%$, which is generic to the volume fraction range 20 to $55 \%$ investigated. It presents the mean value $\langle\rho\rangle$ and the standard deviation $\sigma_{\rho} \equiv \sqrt{\left\langle\rho^{2}\right\rangle-\langle\rho\rangle^{2}}$ of the elongation for strains up to 20 . At $\dot{\gamma} t=20$, the segments have on average lengthened by typically $10^{3}$, which is about one hundred times larger than in the case of a pure liquid. The striking result is that the presence of particles in a shear flow changes the very nature of the stretching laws from linear to exponential. Indeed, the elongation of material line in a simple shear (without particles) follows

$$
\rho_{\text {lin }}(\theta, t)=\sqrt{1+2 \cos \theta \sin \theta \dot{\gamma} t+\sin ^{2} \theta \dot{\gamma}^{2} t^{2}},
$$

where $\theta$ denotes the angle between the line initial orientation and the flow direction. On averaging $\rho_{\text {lin }}^{2}(\theta, t)$ over all possible orientations, we obtain

$$
\rho_{\operatorname{lin}}(t)=\sqrt{1+\dot{\gamma}^{2} t^{2} / 2},
$$

which is only of order 10 for a strain of 20 , and increases linearly with time for large strains. Equation (4.3) is ploted in figure 7 to illustrate the contrast with the elongations actually measured in particulate suspensions: the mean elongation in suspensions is different both in magnitude and in law.

Moreover, by contrast with the pure fluid case, the stretching variability of individual material lines is very broad as evidenced by the exponential growth of the standard deviation $\sigma_{p}$. These results corroborate the preliminary blob experimental visualizations where, in the suspension, many filaments having very different transverse thickness can be observed while the pure fluid case solely exhibits one uniform thickness, (figure 5d). 

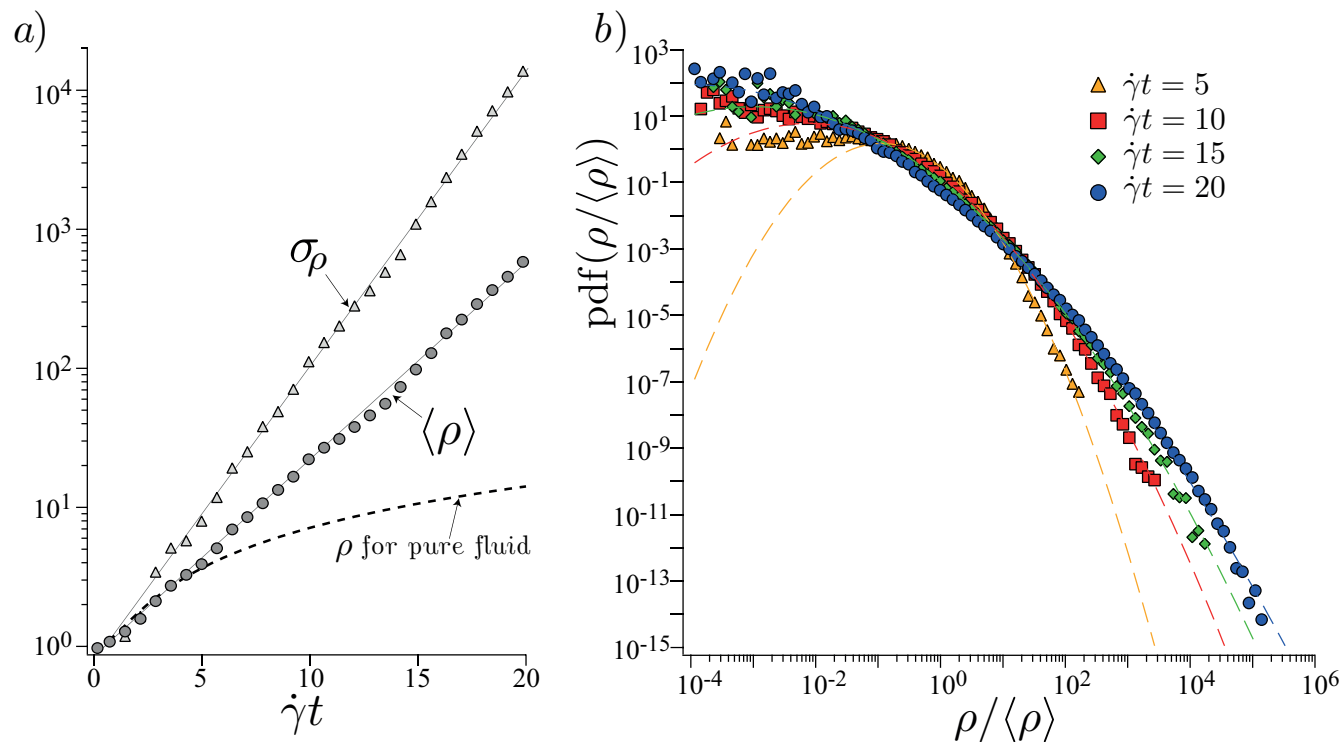

FIGURE 7. Stretching laws measured for a suspension with volume fraction $\phi=35 \%$. a) Mean value $\langle\rho\rangle$ and standard deviation $\sigma_{\rho}=\sqrt{\left\langle\rho^{2}\right\rangle-\langle\rho\rangle^{2}}$ of the distribution of elongations versus macroscopic strain in a semilogarithmic representation. The dashed line corresponds to the mean elongation in a pure fluid $\rho_{\operatorname{lin}}(t)=\sqrt{1+\dot{\gamma}^{2} t^{2} / 2}$. b) Distribution of the normalized elongations $\rho /\langle\rho\rangle$ at different strains. The dashed curves are log-normal distributions built from the mean value $\langle\rho\rangle$ and standard deviation $\sigma_{\rho}$ of the experimental elongation distributions.

More precisely, figure $7 \mathrm{~b}$ shows the distributions of the relative elongations $P(\rho /\langle\rho\rangle)$ at successive strains. The distribution of elongations broadens rapidly such that at a strain $\dot{\gamma} t=20$, it spans more than eight decades. At that strain, the right tail of the distribution contains segments elongated $10^{4}$ times relative to the average $\langle\rho\rangle$, which corresponds to an absolute elongation of $\rho \sim 10^{7}$, in stark contrast with the uniform average elongation of 10 obtained in a simple shear. As figure $7 \mathrm{~b}$ shows, these distributions are found to be well fitted by log-normal distributions (shown as dashed lines). Note that the apparent absence of data on the left hand side of the distributions is fully consistent with lognormal distributions. Indeed, for broad distributions, the statistical weight of the left hand side of the distribution vanishes. Our data thus fully resolve the meaningful part of the distribution.

The advective strip method presented above was repeated with velocity fields measured in suspensions with different volume fractions $\phi$ ranging from $20 \%$ to $55 \%$. The same trends as those detailed for $\phi=35 \%$ are systematically observed. As shown in figure 8 , it is moreover found that larger particulate volume fractions increase both the growth rate of the average elongation $\langle\rho\rangle$ and that of the standard deviation $\sigma_{\rho}$. This indicates that a larger volume fraction results in larger fluid disturbances which, in turn, induce a faster and more random elongation of the fluid material lines. Fitting these curves with exponential growths in strain $\mathrm{e}^{\kappa \dot{\gamma} t}$ yields for $\langle\rho\rangle, \kappa_{\rho}=0.09+0.74 \phi$, and for $\sigma_{\rho}$, $\kappa_{\sigma_{\rho}}=0.12+1.03 \phi$. In the range of volume fraction investigated, the growth rates are found to increase linearly with $\phi$. No measurements could be performed above $55 \%$ as the large normal stress built in the suspension starts to deform the belt.

To summarize, by kinematically advecting passive segments using the experimental velocity fields of the fluid phase, we measured the elongation of fluid material lines in sheared particulate suspensions. Two important features characterize these elongations: 
a)

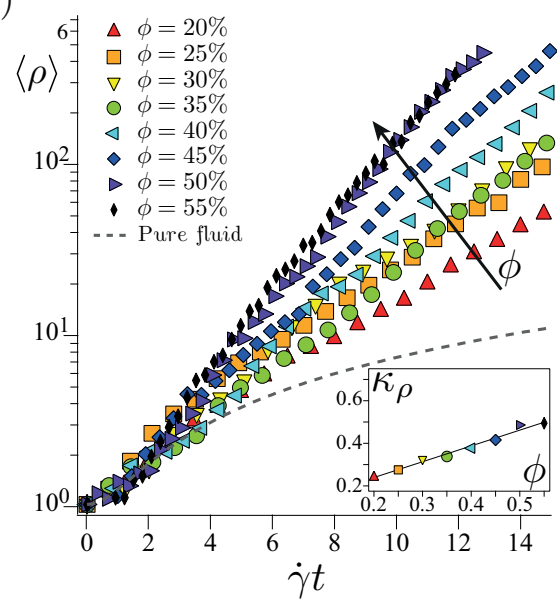

b)

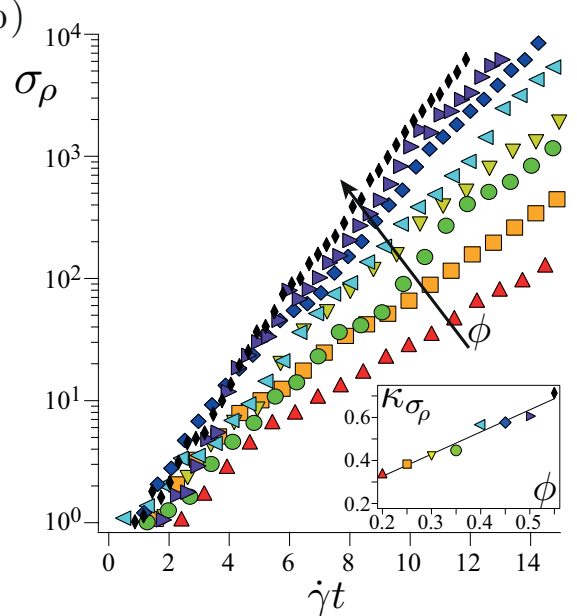

Figure 8. Mean elongation $\langle\rho\rangle$ (a) and standard deviation $\sigma_{\rho}$ (b) versus strain for increasing volume fractions ranging from 20 to $55 \%$. Insets: growth rate $\kappa$ of the exponential fit $\mathrm{e}^{\kappa \dot{\gamma} t}$ to the main curves, as a function of $\phi$. The lines show $\kappa_{\rho}=0.09+0.74 \phi(\mathrm{a})$, and $\kappa_{\sigma_{\rho}}=0.12+1.03 \phi$ (b).

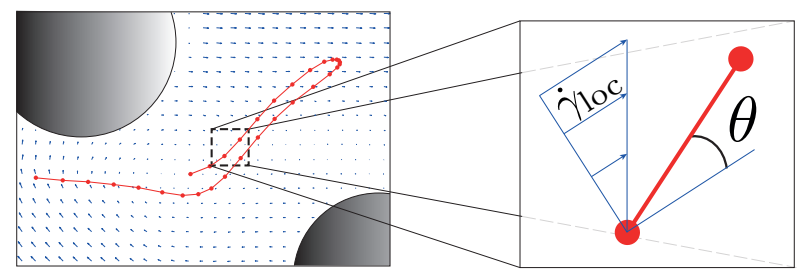

FiguRE 9. Each segment composing a fluid material line is locally stretched by a simple shear $\dot{\gamma}_{\text {loc }}$ which is oriented with a random angle $\theta$ with respect to the segment.

i) the mean and the standard deviation grow exponentially, ii) the distribution converges to a log-normal. In the following, using two measurable properties of the fluid velocity field, namely the local shear rate distribution and the Lagrangian correlation time, we present a mechanism accounting for these observations.

\section{Origin of the stretching laws}

\subsection{Principle}

We consider the elementary component of a fluid material line: the segment, see figure 9. At that scale, much smaller than the particle size, the local shear $\dot{\gamma}_{\text {loc }}$ is uniform. Considering the broad distribution of the segment orientations, we assume that the local shear rate has a random orientation with respect to the segment. Therefore, as long as the local shear rate $\dot{\gamma}_{\text {loc }}$ persists, the average elongation of the segment is (see equation $4.3)$

$$
\rho=\sqrt{1+\dot{\gamma}_{\text {loc }}^{2} t^{2} / 2}
$$

Note that an individual segment can be stretched or compressed depending on wether it is located in a diverging or compressive region of the flow, respectively. However, once averaged over all possible orientations, the segment net elongation is strictly larger than unity. Two questions then naturally emerge: what are the local shear rates? and how long 

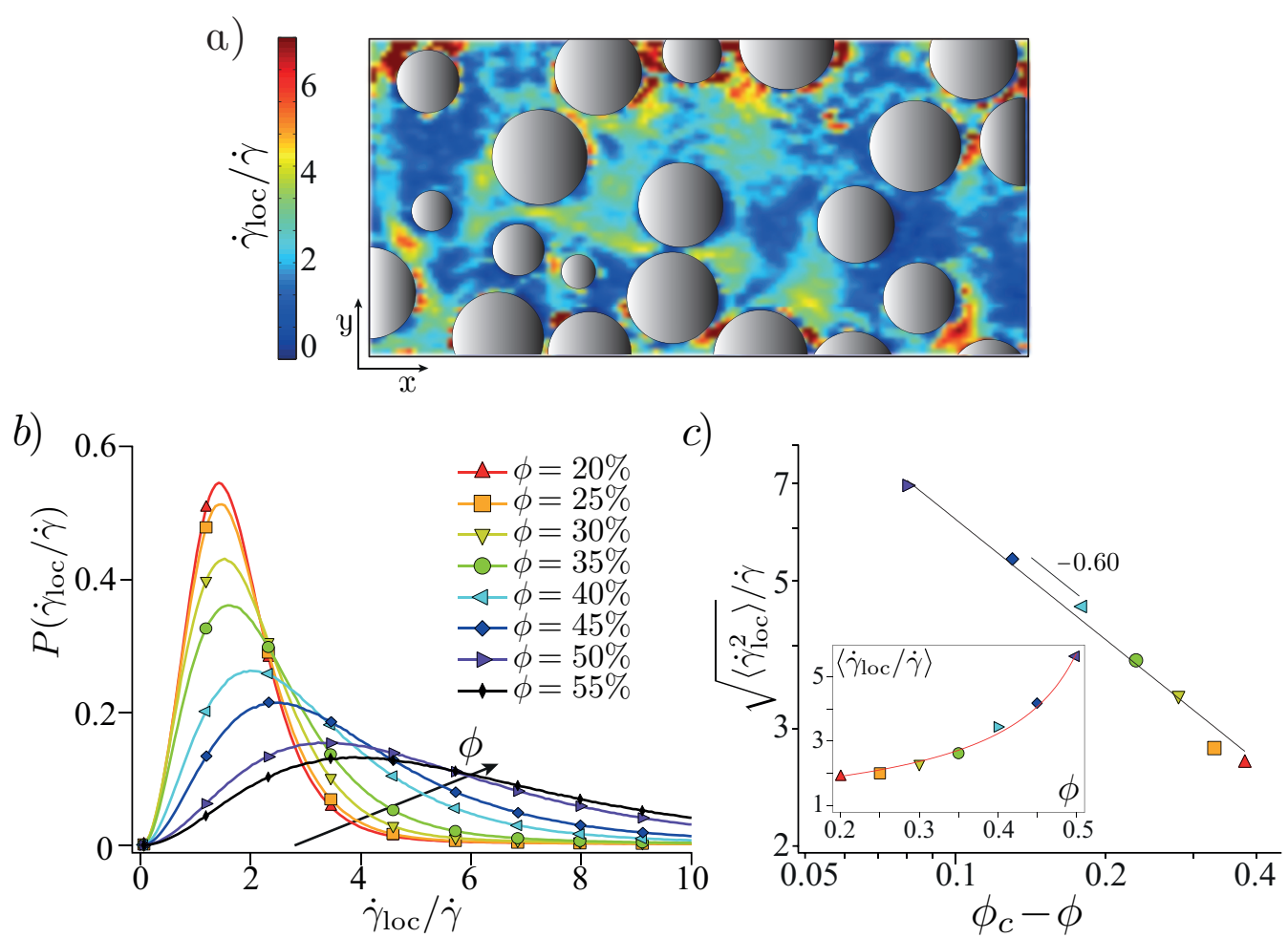

Figure 10. a) Typical local shear rate map for a suspension with volume fraction $\phi=35 \%$. b) Experimental distributions of normalized local shear rate $P\left(\dot{\gamma}_{\text {loc }} / \dot{\gamma}\right)$ for different volume fractions (the solid line is not a fit but the experimental data, sparse markers are used for sake of clarity). c) $\sqrt{\left\langle\dot{\gamma}_{\text {loc }}^{2}\right\rangle} / \dot{\gamma}$ versus $\phi_{\mathrm{c}}-\phi$. The best fit $\sqrt{\left\langle\dot{\gamma}_{\text {loc }}^{2}\right\rangle} / \dot{\gamma} \sim\left(\phi_{\mathrm{c}}-\phi\right)^{-\beta}$, with $\phi_{\mathrm{c}}=0.58$, yields $\beta=0.601$ (see text). Inset: mean normalized local shear rate $\left\langle\dot{\gamma}_{\text {loc }} / \dot{\gamma}\right\rangle$ versus $\phi$. The line is the best fit by $A /\left(\phi_{\mathrm{c}}-\phi\right)^{\delta}$.

do these shear rates persist? In the following two sections, we address these questions by providing information about the local shear rates and the Lagrangian correlation time of the velocity field.

\subsubsection{Local shear rate}

We measure the local shear rate from the experimental two-dimensional velocity fields. To this end, we define the local shear rate by the norm of the symmetric part of the strain tensor:

$$
\dot{\gamma}_{\mathrm{loc}}=\sqrt{2\left(\frac{\partial u}{\partial x}\right)^{2}+2\left(\frac{\partial v}{\partial y}\right)^{2}+\left(\frac{\partial u}{\partial y}+\frac{\partial v}{\partial x}\right)^{2}},
$$

where $\{u, v\}$ are the $\{x, y\}$ components of the velocity field. This definition disregards the rotation part of the strain tensor. For a simple shear, one has $\dot{\gamma}_{\text {loc }}=$ cte $=\dot{\gamma}$. Figure 10a shows a typical local shear rate map, obtained in a suspension with volume fraction $\phi=35 \%$. The color-scale represents $\dot{\gamma}_{\text {loc }}$ amplitude normalized by the applied macroscopic shear rate $\dot{\gamma}$, that is to say the amplification of the shear due to the presence of particles. The local shear rate is highly non-uniform and its value can greatly exceed the macroscopic shear rate. Interestingly, large local shear rates occur preferentially in 

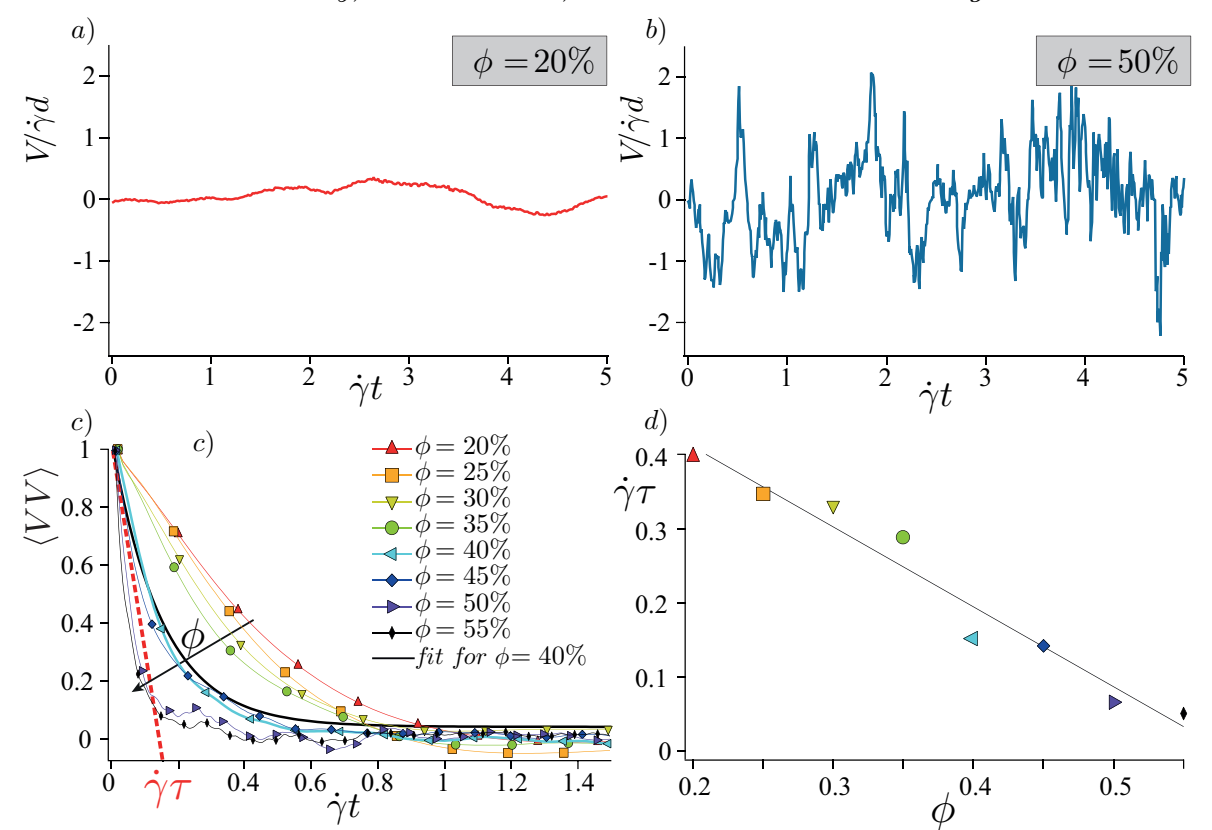

FIGURE 11. (a-b) Lagrangian velocity transverse to the flow of a tracer passively advected by the suspending liquid $V$, as a function of the strain $\dot{\gamma} t$. a) $\phi=20 \%$ and b) $\phi=50 \%$. c) Average Lagrangian velocity auto-correlation function $\langle V V\rangle$ obtained for different volume fractions versus strain. The velocity auto-correlation functions fit well $\mathrm{e}^{-\dot{\gamma} t / \dot{\gamma} \tau}$ where $\tau$ denotes the correlation time. d) Correlation strain $\dot{\gamma} \tau$ versus $\phi$ and corresponding linear fit $\dot{\gamma} \tau=0.62-1.08 \phi$.

the vicinity of the particles, however there is no apparent correlation between large local shear rates and small inter-particle distances.

More quantitatively, we report in figure 10b the distribution of normalized local shear rates obtained for various volume fractions. Clearly, the local shear rate exceeds most of the time the imposed macroscopic shear rate, sometimes by one order of magnitude, and this trend accentuates with increasing volume fractions. The mean normalized value $\left\langle\dot{\gamma}_{\text {loc }}\right\rangle / \dot{\gamma}$ is plotted versus $\phi$ in the inset of figure 10c. It is found to fit well $\left\langle\dot{\gamma}_{\text {loc }}\right\rangle / \dot{\gamma}=$ $A /\left(\phi_{\mathrm{c}}-\phi\right)^{\delta}$. Fixing $\phi_{\mathrm{c}}=0.58$ this yields $A \simeq 0.56$ and $\delta \simeq 0.7$. Note that the last point, corresponding to $\phi=55 \%$, was not included in the fitting procedure since we suspect that it is biased by the deflexion of the belt mentioned above. Note also that PIV using smaller boxes resulted in very similar local shear rate distributions with less than $6 \%$ difference on the average.

The trends discussed above may also be interpreted in terms of a macroscopic viscosity. In such case, the relevant quantity to investigate is the second moment of the local shear rate distribution $\left\langle\dot{\gamma}_{\text {loc }}^{2}\right\rangle$ (Chateau 2008, Lerner 2012, Dagois-Bogy 2015). Values of this quantity have recently been obtained by Trulson et al. from numerical simulations of dense frictional suspensions (Trulsson 2016). They report that $\sqrt{\left\langle\dot{\gamma}_{\text {loc }}^{2}\right\rangle} / \dot{\gamma} \sim(J / \mu)^{-1 / 3}$, where $J=\dot{\gamma} \eta_{f} / P$ is the viscous number, with $P$ the confining pressure, and $\mu$ the suspension macroscopic friction coefficient. Since $\eta_{s} / \eta_{f}=\sigma / \dot{\gamma} \eta_{f}=\mu / J$, this results in $\sqrt{\left\langle\dot{\gamma}_{\text {loc }}^{2}\right\rangle} / \dot{\gamma} \sim\left(\eta_{s} / \eta_{f}\right)^{1 / 3}$. Combining this with $\eta_{s} / \eta_{f} \sim\left(\phi_{c}-\phi\right)^{-2}$ and using $\phi_{c}=0.58$ (Boyer 2011) leads to $\sqrt{\left\langle\dot{\gamma}_{\text {loc }}^{2}\right\rangle} / \dot{\gamma} \sim\left(\phi_{c}-\phi\right)^{-2 / 3}$, which is in fairly good agreement with the measured scaling, as figure 10c shows. 


\subsubsection{Lagrangian correlation time}

The second important quantity of the suspending liquid flow is the persistence time of the velocity fluctuations induced by the particles. Figures 11a and b show the transverse Lagrangian velocity $V$ (perpendicular to the flow) of a passive tracer advected by the fluid at a low and a large volume fraction, respectively. Consistently with the magnitude of the local shear, more concentrated particulate suspensions develop velocity fluctuations with larger amplitudes. However these fluctuations are found to persist on a much shorter time as $\phi$ increases. The duration $t_{\mathrm{c}}$ for which a segment is coherently stretched by the flow is directly prescribed by this persistence time, which we define from the Lagrangian velocity auto-correlation functions. As shown in figure 11c, these functions decorrelate exponentially with strain. In the range of volume fraction investigated, the dimensionless correlation time $\dot{\gamma} \tau$, inferred from this exponential decay, decreases linearly with $\phi$ as

$$
\dot{\gamma} \tau \simeq 0.62-1.08 \phi
$$

(see figure 11d). We expect $t_{\mathrm{c}}$ to be of the order of $\tau$ and thus write

$$
t_{\mathrm{c}}=\alpha \tau,
$$

with $\alpha$ an order one constant. Note that as shown in figure 7 , this persistence time $\left(\lesssim \dot{\gamma}^{-1}\right)$ is much shorter than the observation period $\left(\gtrsim 10 \dot{\gamma}^{-1}\right)$.

\subsubsection{Multiplicative stretching process}

With informations about the local shear rates and their persistence time at hand, we now explain the elongations of fluid material lines as a sequence of uncorrelated cycles of stretching. During the first cycle of duration $t_{c}$, a given segment of a material line is elongated by the local shear rate $\dot{\gamma}_{\text {loc }, 1}$ resulting in a stretching

$$
\Delta \rho_{1}=\sqrt{1+\left(\dot{\gamma}_{\text {loc }, 1} t_{\mathrm{c}}\right)^{2} / 2},
$$

where $\dot{\gamma}_{\text {loc,1 }}$ is a local shear rate which probability is prescribed by the distribution $P\left(\dot{\gamma}_{\text {loc }} / \dot{\gamma}\right)$, cf. figure $10 \mathrm{~b}$. After the duration $t_{\mathrm{c}}$, the local velocity field de-correlates and the local shear rate map is entirely redistributed. The segment then experiences a new local shear rate $\dot{\gamma}_{\mathrm{loc}, 2}$, which at $t=2 t_{\mathrm{c}}$ yields $\rho=\Delta \rho_{1} \sqrt{1+\left(\dot{\gamma}_{\mathrm{loc}, 2} t_{\mathrm{c}}\right)^{2} / 2}$, and so on. The total elongation at time $t$, after $N=t / t_{c}$ cycles, is the product of all the elementary elongations occurring at each cycle $\rho(t)=\prod_{i=1}^{N=t / t_{c}} \Delta \rho_{i}$. The logarithm of this expression can be written as a sum

$$
\ln \rho \equiv \sum_{i=1}^{t / t_{\mathrm{c}}} \ln \Delta \rho_{i}=\frac{1}{2} \sum_{i=1}^{t / t_{\mathrm{c}}} \ln \left[1+\left(\dot{\gamma}_{\mathrm{loc}, i} t_{\mathrm{c}}\right)^{2} / 2\right] .
$$

Since the elementary stretchings are independent, the distribution of $\ln \rho$ is expected, by virtue of the central limit theorem, to be normal. This multiplicative stretching model thus predicts $\rho$ to converge, after a few $t / t_{c}$ cycles, to a log-normal distribution. This prediction is in agreement with the experimental results shown in figure $7 \mathrm{~b}$. The distribution of $\ln \rho$, i.e. the normal distribution, writes

$$
P(x=\ln \rho)=\frac{1}{\sqrt{2 \pi} \sigma} \mathrm{e}^{-\frac{(x-\mu)^{2}}{2 \sigma^{2}}},
$$

with a non-zero mean

$$
\mu \equiv\langle\ln \rho\rangle=\frac{\langle\ln \Delta \rho\rangle}{\dot{\gamma} t_{\mathrm{c}}} \dot{\gamma} t
$$


and variance

$$
\sigma^{2} \equiv\left\langle\ln ^{2} \rho\right\rangle-\mu^{2}=\frac{\left\langle\ln ^{2} \Delta \rho\right\rangle-\langle\ln \Delta \rho\rangle^{2}}{\dot{\gamma} t_{\mathrm{c}}} \dot{\gamma} t
$$

Both the mean and the variance of the distribution of $\ln \rho$ increase linearly with time. They also vary with the particulate volume fraction due to the $\phi$-dependence of $\dot{\gamma}_{\text {loc }}$ and $t_{\mathrm{c}}$. This variation with $\phi$ is better appreciated by recasting equations (5.8) and (5.9) into

$$
\begin{gathered}
\mu=f(\phi) \dot{\gamma} t, \\
\sigma^{2}=g(\phi) \dot{\gamma} t,
\end{gathered}
$$

with $f(\phi) \equiv\langle\ln \Delta \rho\rangle / \dot{\gamma} t_{\mathrm{c}}$ and $g(\phi) \equiv\left(\left\langle\ln ^{2} \Delta \rho\right\rangle-\langle\ln \Delta \rho\rangle^{2}\right) / \dot{\gamma} t_{\mathrm{c}}$ only depending on $\phi$. Note that $f(\phi)$ and $g(\phi)$ are crucial quantities. Since the time dependency is known, they contain all the information about the asymptotic of the stretching laws in suspensions.

The multiplicative stretching model not only explains the origin of the log-normal distributions of elongations measured experimentally, but also the exponential increase of the mean elongation $\rho$ and variance $\sigma_{\rho}^{2}$ shown in figure 7. Indeed, the mean and variance of the (log-normal) distribution of $\rho$ can be deduced from the mean and the variance of the (normal) distribution of $\ln \rho$ following

$$
\langle\rho\rangle=\mathrm{e}^{(f+g / 2) \dot{\gamma} t},
$$

and

$$
\sigma_{\rho}^{2}=\left(\mathrm{e}^{g \dot{\gamma} t}-1\right) \mathrm{e}^{(2 f+g) \dot{\gamma} t} \simeq \mathrm{e}^{2(f+g) \dot{\gamma} t},
$$

the last simplification in $\sigma_{\rho}^{2}$ becoming true after a few $t_{\mathrm{c}}$.

Furthermore, the particulate volume fraction dependence of $f(\phi)$ and $g(\phi)$ can be computed from the persistence time $t_{\mathrm{c}}$ and the distribution of local shear rates, using equations (5.10) and (5.11) together with equations (5.8) and (5.9). In the experimental range $20 \%<\phi<55 \%$, this yields

$$
f(\phi) \simeq 0.104+0.298 \phi,
$$

and

$$
g(\phi) \simeq-0.069+0.810 \phi,
$$

with the structure constant $\alpha$, set once and for all $\phi$, to 0.3 for computing $\mu$, and to 3.9 for computing $\sigma^{2}$. These rates $f$ and $g$ both increase with $\phi$, in agreement with the experimental trends. Note that this dependence on the volume fraction is non-trivial, since $f$ and $g$ result from the product of $\dot{\gamma}_{\text {loc }}$ and $t_{\mathrm{c}}$, which have opposite trends with $\phi$ : the former increases whereas the latter decreases with increasing $\phi$.

The predictions of the multiplicative stretching model are compared to the experimental stretching laws obtained by the Diffusive Strip Method in figure 12. The agreement is good for all volume fractions and all strains, which suggests that the multiplicative stretching model presented above captures the relevant mechanisms at the origin of the stretching laws.

\subsection{Comments on the stretching process}

Stretching of material elements in nature, may they be passive like in the present case, or with internal restoration forces like polymers (Shaqfeh 1992, Afonso 2005) may have different origins. The stochastic models to describe them usually present a net drift, and a random noise terms. The relative amplitudes of these two contributions are in our analysis given by $f(\phi)$ and $g(\phi)$, respectively (see equations (5.10) and (5.11)). The first term sets the growth of $\langle\ln \rho\rangle$, while the second sets that of $\left\langle\ln ^{2} \rho\right\rangle-\langle\ln \rho\rangle^{2}$. At a 

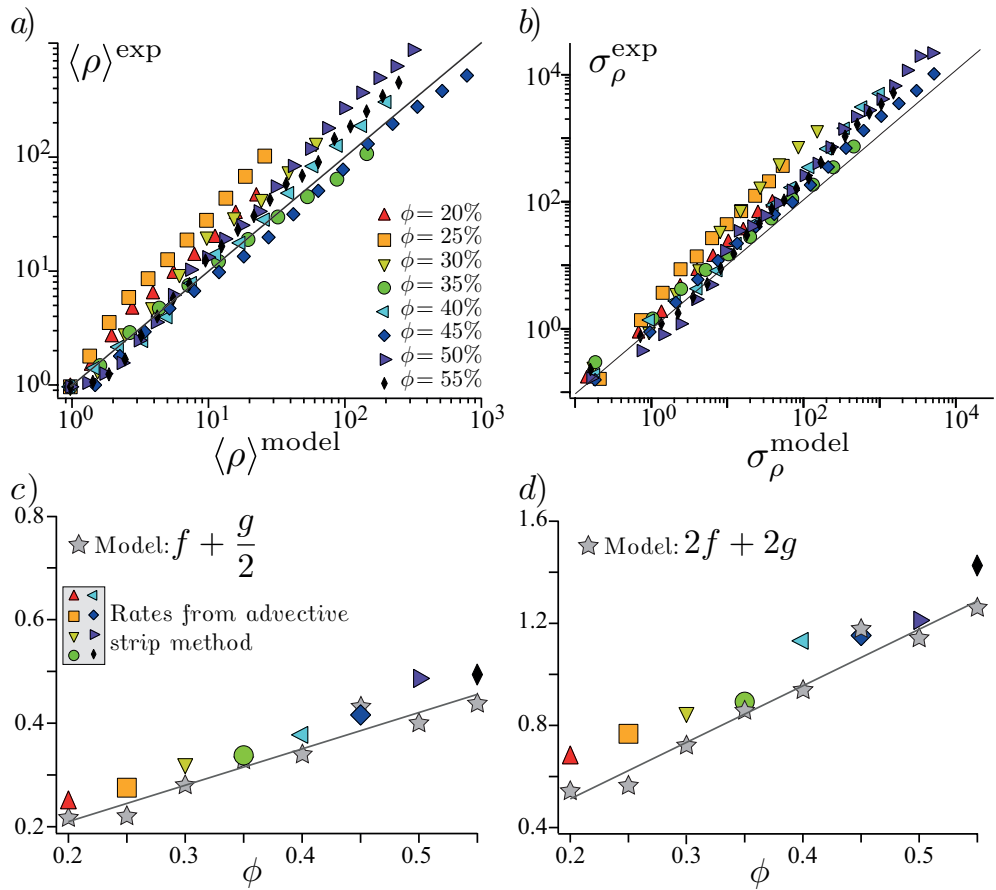

FiguRE 12. Comparison between the experimental stretching (extracted from figure 8) and the multiplicative stretching model. (a-b) Mean elongation $\langle\rho\rangle$ and standard deviation $\sigma_{\rho}$. The values from the advective strip method are plotted versus those predicted by the multiplicative stretching model. (c) Comparison between the exponential rates of the mean elongation $\kappa_{\rho}$ obtained from the advective strip method (see figure 8a) and the model prediction $f+g / 2$ (5.12). (d) Comparison between the exponential rates of the variance of the elongation $2 \kappa_{\sigma_{\rho}}$ (see figure $8 \mathrm{~b})$ and the model prediction $2(f+g)(5.13)$.

microscopic level, the growth of a given material line depends on its orientation with that of the local velocity gradient. The line length $l(t)$ may increase or decrease depending on wether it is aligned with a diverging or compressive region of the flow. For instance, in a flow corresponding to the pure Brownian motion limit (Cocke 1971), for which $\dot{\rho} / \rho=B(t)$ with $B(t)$ a zero-mean, Delta correlated noise, i.e. $\langle B(t)\rangle=0$ and $\left\langle B\left(t^{\prime}\right) B\left(t^{\prime \prime}\right)\right\rangle=\left(1 / \tau_{0}\right) \delta\left(t^{\prime}-t^{\prime \prime}\right)$, these two contributions are balanced and the net line growth $\mathrm{d} \ln \rho(t) / \mathrm{d} t=l(\dot{t}) / l(t)$ is, on averaging over all directions, identically zero. In that case, representative of $t_{\mathrm{c}} \rightarrow 0$, the material lines only grow through the contribution of the fluctuations of $B(t)$, which results in $\mathrm{d}\langle\ln \rho\rangle / \mathrm{d} t=0$, and $\left\langle(\ln \rho)^{2}\right\rangle \sim 2 t / \tau_{0}$ : the logarithm of the elongation diffuses.

In particulate suspensions, the situation is different since if the direction of the stretch indeed changes at random, it has a finite persistence time. In such case, it has been shown that material lines tend to preferentially align in the direction of elongations (see Cocke 1969, Orszag 1970, Girimaji 1990, Duplat 2000 and also equation 5.5). Thus, over an observation period larger than the (non-zero) correlation time $t_{\mathrm{c}}$, we expect

$$
\frac{\mathrm{d}}{\mathrm{d} t}\langle\ln \rho\rangle>0 .
$$

This agrees with our measurements shown in figure 13(a): the finite persistence time of the stretching field results in a positive drift term: $f(\phi)>0$. Although some material lines are subject to compression (see the negative values on the distribution of 

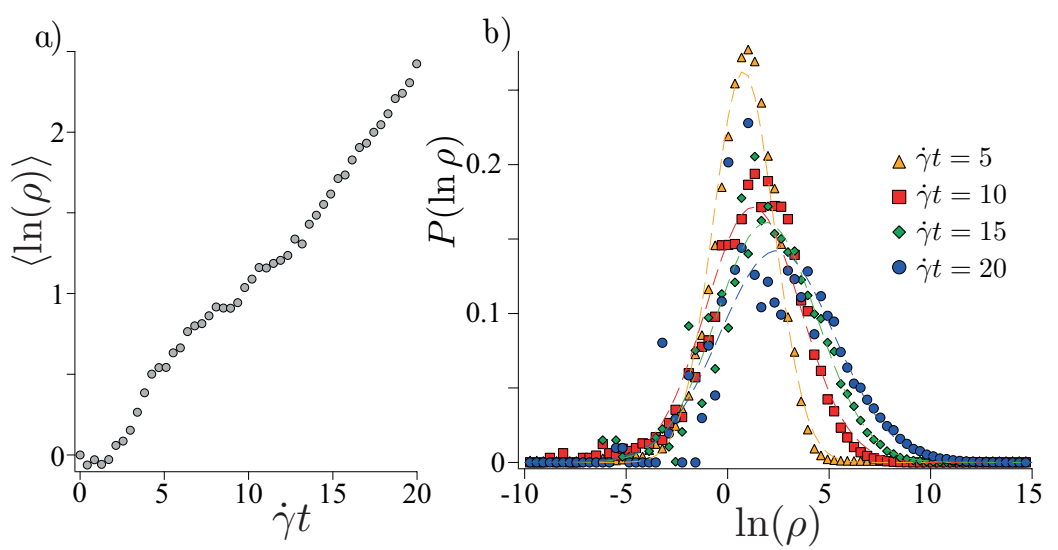

Figure 13. a) Mean logarithm of the material line elongations, $\langle\ln \rho\rangle$ versus $\dot{\gamma} t$ for a suspension of volume fraction $\phi=35 \%$. b) PDF of the logarithm of the material line elongations $P(\ln \rho)$ at successive times.

$\ln \rho$ in figure 13(b)), on average, the logarithm of the elongations $\langle\ln \rho\rangle$ increases with time. Material lines in particulate suspensions thus grow from the contribution of both a drift and a noise. The stretching process thus corresponds to a noisy multiplicative sequence of correlated motions, like the random Sine Flow (Meunier 2010), or porous media flows (Le Borgne 2015). Porous media and sheared particulate suspensions have similar exponential stretching laws. This is true in $3 \mathrm{D}$ systems as in both cases the fluid trajectories are chaotic. Note however that for $2 \mathrm{D}$ systems the implications of steadiness change the picture qualitatively. In a $2 \mathrm{D}$ porous media, the flow is steady and there are only two degrees of freedom : the flow is thus not chaotic. The elongation of material lines in 2D synthetic porous media have been shown to grow algebraically rather than exponentially (Le Borgne 2015). Conversely in 2D sheared suspensions, the time dependence of the flow allows the system to be chaotic (Metzger 2013). One therefore expect to observe exponential stretching laws in sheared particulate suspensions also in purely $2 \mathrm{D}$ configurations.

\subsection{Further remarks}

We would like to point out certain limitations of the present study. First, the present findings and their analysis are restricted to the particulate volume fraction $20 \% \leqslant \phi \leqslant$ $55 \%$, for which material lines in the suspending liquid stretch exponentially with strain. This is not necessarily the case outside form this range. In particular, as $\phi \rightarrow 0$, this exponential trend must cross-over to linear since the elongation of material lines in a simple shear is linear with strain. We however anticipate that the exponential trend could hold down to fairly low volume fractions but only emerge after increasingly large strains, since the velocity correlation time in the dilute limit should follow $\tau \sim(\dot{\gamma} \phi)^{-1}$ and diverge at low $\phi$. Further investigations are needed to characterize this dilute regime $(\phi<20 \%)$.

Second, the PIV measurements performed here are two-dimensional and provide the fluid velocity projected in the $(x y)$ plane only. They therefore neglect part of the stretching of the material lines, namely that involving deformations in the vorticity direction $(z)$. However, we believe that they resolve the stretching mechanism and most of its magnitude for the following reasons: i) these measurements resolve the fluid displacements in the gradient direction $(y)$, which is the only direction for which displacements couple with the main shear flow to produce an enhance stretching. The fluctuations in the vorticity 


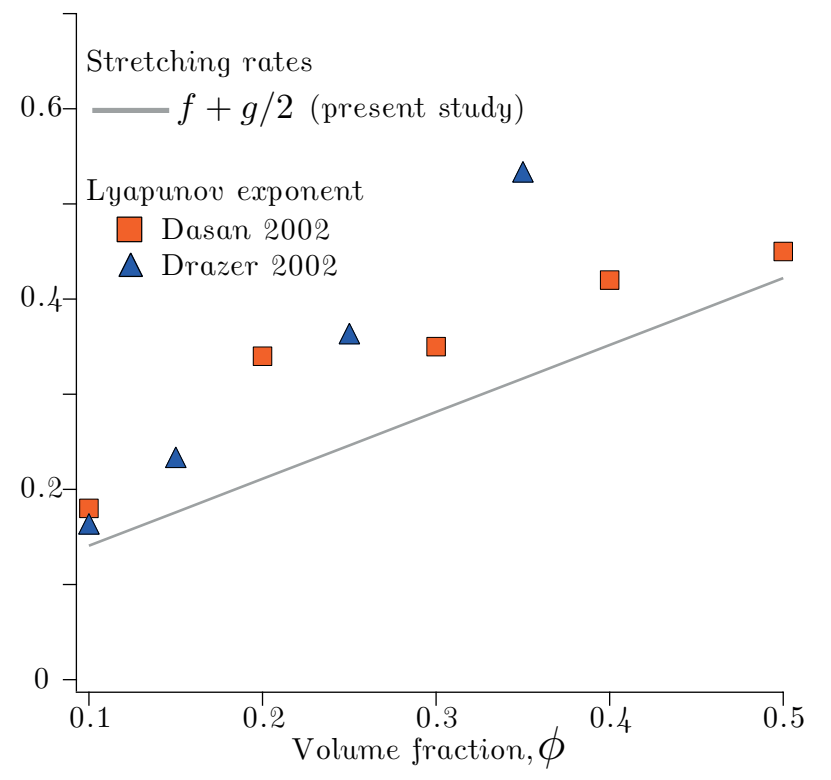

FIGURE 14. Comparison between the stretching rates obtained in the present study and the largest Lyapunov exponent obtained from 3D Stokesian dynamics simulations (Dasan 2002, Drazer 2002).

direction are thus expected to produce less stretching than those occurring in the gradient direction. ii) Particles in a shear flow rotate mainly about the vorticity axis thereby inducing fluid disturbances mostly in the velocity-gradient plane, which we consider. Here again, the effects of the velocity disturbances induced by the particle rotation should be smaller in the vorticity direction than those occurring in the velocity-gradient plane. iii) More quantitatively, the stretching rates $f+g / 2$ predicted by the present model based on 2D data are in good agreement with the largest Lyapunov exponents obtained from 3D Stokesian simulations, see figure 14. From the above considerations, it is likely that the mechanisms at the origin of the scalar dispersion, stretching and subsequent mixing are well characterized by the present measurements, even though those are limited to the information contained in the $x y$ plane.

Third, as already mentioned in section 3 , the stretching of material lines is exponential at every scale, but the stretching of a material blob with thickness $s_{0}$ is expected to follow that of material lines only if its thickness is smaller than the correlation scale of the fluid motion, which is of order $d$ (in the other case, the blob is first essentially stretched by the macroscopic shear $\dot{\gamma}$ until $s \leqslant d$ ). In the following, we will therefore only consider the relevant case $s_{0} \leqslant d$.

The latter considerations have important consequences on the estimation of the blob thickness $s$, hence on the mixing time that we will address in the next section. For an arbitrary elongation $w / w_{0}$ in the vorticity direction $(z)$, mass conservation gives $s_{0} l_{0} w_{0}=$ $s(t) l(t) w(t)$. However, in light of the above discussion, the flow is assumed to be two dimensional with $w / w_{0} \ll l / l_{0}$. Mass conservation thus results in

$$
s_{0} l_{0}=s(t) l(t)
$$

Using direct image analysis, we have checked that this is experimentally verified. A blob with initial surface $s_{0} l_{0}$ being converted into a strip with length $l(t)$ and thickness $s(t)$ 
a)

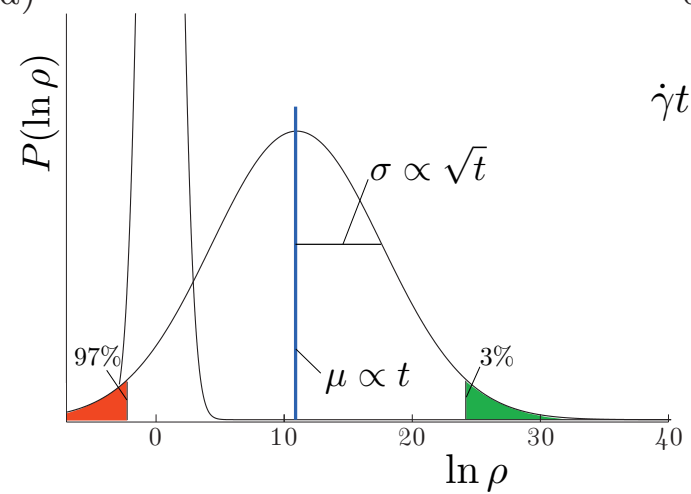

b)

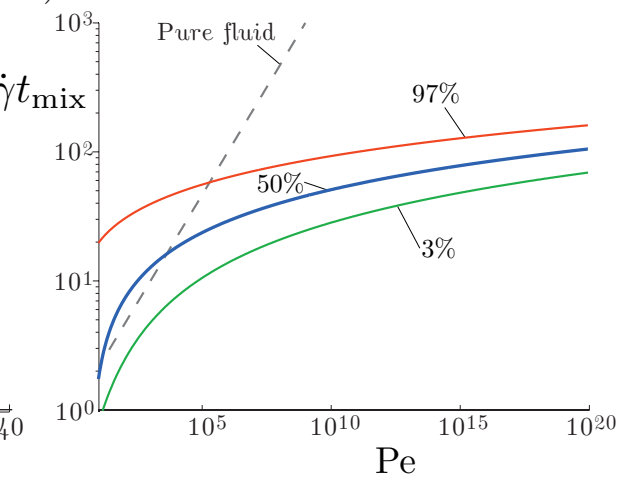

Figure 15. a) Evolution of the distribution $P(\ln \rho)$ of the logarithm of the material line elongations in a particulate suspension. The mean of the distribution $\mu \sim t$ and its standard deviation $\sigma \sim \sqrt{t}$. b) Dimensionless mixing times $\dot{\gamma} t_{\text {mix }}$ in a suspension $(\phi=35 \%)$ as a function of Pe. The median (blue line), the most stretched $t_{\text {mix }}^{3 \%}$ (green line), and the less stretched $t_{\text {mix }}^{97 \%}$ (red line) dimensionless mixing times can be compared to the dimensionless mixing time $\sim P e^{1 / 3}$ expected in a pure fluid (dashed line).

indeed obeys, before it starts mixing, to equation (5.17), suggesting that the flow is indeed area preserving.

\section{Implications for mixing}

In such area preserving flow, the thickness $s(t)$ of a distorted blob decreases in inverse proportion of its length $l(t)$ according to equation (5.17). As recalled in the introduction, the mixing time for a given blob portion of thickness $s$ is reached when its compression rate $-\dot{s} / s$ is balanced by its rate of diffusive broadening $D / s^{2}$. At that time, called the mixing time, the scalar concentration carried by that portion of the blob starts to significantly decay i.e., mix. Since in particulate suspensions $\rho=l(t) / l_{0}=e^{\kappa \dot{\gamma} t}$, the mixing time writes $t_{\text {mix }} \simeq \dot{\gamma}^{-1} \ln (\kappa P e) /(2 \kappa)$.

We also found that the logarithm of the elongations of an ensemble of such material lines is normally distributed with a mean and a variance growing linearly with time following $\mu=\langle\ln \rho\rangle=f(\phi) \dot{\gamma} t$ and $\sigma^{2}=g(\phi) \dot{\gamma} t$ (see equations (5.10) and (5.11), respectively). These results are illustrated in figure $15 \mathrm{a}$. Since, similarly to the logarithm of the elongations, the stretching rates, $\kappa \dot{\gamma}=\ln \rho / t$ are normally distributed, the median mixing time, obtained for the mean stretching rate, i.e. for $\langle\kappa \dot{\gamma}\rangle=\langle\ln \rho\rangle / t=\mu / t=f(\phi) \dot{\gamma}$, is

$$
t_{\mathrm{mix}}^{\mathrm{med}} \approx \frac{1}{2 f(\phi) \dot{\gamma}} \ln (f(\phi) P e)
$$

Considering a blob distorted in such a way that it samples all the possible elongations in the global statistics, the above estimate provides the time at which half of the blob has reached its mixing time. The logarithmic dependence of the mixing time on the Péclet number is different from that obtained in a simple shear flow (without particles) for which $\rho \simeq \dot{\gamma} t$ yields $t_{\text {mix }} \simeq \dot{\gamma}^{-1} P e^{1 / 3}$. Introducing particles in a viscous fluid therefore becomes more and more efficient at reducing the mixing time as the Péclet number increases. In the present study, the Péclet number is $P e \sim 10^{6}$. The median mixing time for $\phi=35 \%$ is thus $t_{\text {mix }}^{\text {med }} \simeq 30 / \dot{\gamma}$, which has to be compared with $t_{\text {mix }} \simeq 100 / \dot{\gamma}$ in a pure shear flow. 


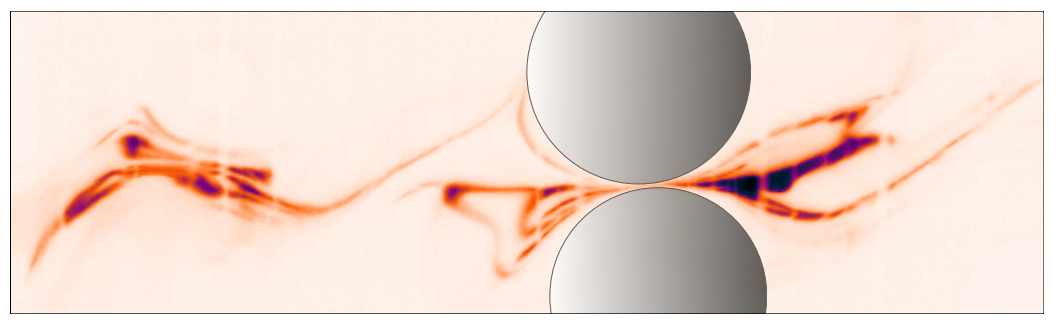

FigURE 16. Picture illustrating the complexity of folding of the stretched blob of dye and the potential interaction (merging) of nearby filaments.

Note that varying the volume fraction from $20 \%$ to $55 \%$ increases $f(\phi)$ only by a typical factor of 2 , which decreases the median mixing time by about the same moderate factor.

In practical situations, mixing half of the scalar may not be the relevant question, precisely because in particulate suspensions, elongations are, as seen in figure 5 , broadly distributed. So are mixing times. To address this point, we estimate, for the same conditions as previously, the mixing times for the portions of the blob that undergo the largest and the lowest stretching rates respectively, i.e. the mixing times corresponding to both tails of the distribution (highlighted in figure 15a). The $3 \%$ most strongly stretched portions of the blob are bounded by $\ln \rho=\mu+2 \sigma$. The expression $-\dot{s} / s=D / s^{2}$ results in $2 f(\phi) \dot{\gamma} t+4 \sqrt{g(\phi) \dot{\gamma} t}=\ln [(f(\phi)+\sqrt{g(\phi) / \dot{\gamma} t}) P e]$, which yields the mean field mixing time $t_{\text {mix }}^{3 \%} \simeq 14 / \dot{\gamma}$. On the other end of the distribution, the less stretched portions of the blob, bounded by $\ln \rho=\mu-2 \sigma$, reach their mixing time at $t_{\text {mix }}^{97 \%} \simeq 64 / \dot{\gamma}$, later than if it were sheared in a pure fluid. In figure $15 \mathrm{~b}$, the median (blue line), the most stretched $t_{\text {mix }}^{3 \%}$ (green line), and the less stretched $t_{\text {mix }}^{97 \%}$ (red line) dimensionless mixing times are plotted as a function of the Péclet number. This shows that if the concern is to mix essentially all the scalar, large Péclet numbers $\left(\gg 10^{5}\right)$ are required before mixing in a suspension becomes more efficient than in a pure fluid. Persistent poorly stretched regions are deterring. The relative width $\sigma / \mu$ of the stretching rate distribution decreases in time like $t^{-1 / 2}$ but this only mildly decreases the spreading of the mixing times as $P e$ increases, since $t_{\text {mix }} \propto \ln P e$. For instance, at $P e=10^{20}$, the mixing times remain fairly distributed with $t_{\text {mix }}^{97 \%} / t_{\text {mix }}^{3 \%}>2$.

Finally, the results obtained on the stretching laws must be related to the overall dispersion of the blob. In a random flow, line stretching, and dispersion, are two different things: because the extent of the area occupied by the blob grows more slowly than the area where the scalar constitutive of the blob is dispersed, the blob will at some point unavoidably reconnect and merge by overlapping onto itself (Duplat 2008). Let us see how: after the mixing time, a scalar blob with length growing like $l(t)=l_{0} e^{\dot{\gamma} t}$ has a transverse concentration profile whose width is confined to the Batchelor scale $\sqrt{D t}$. The area $\mathcal{A}$ occupied by the scalar is thus $\mathcal{A}=\sqrt{D t} l_{0} e^{\dot{\gamma} t}$, growing exponentially in time. Now, the spatial support of the blob undergoes a dispersion induced by the particle effective dispersion coefficient $D_{\text {eff }} \sim \dot{\gamma} d^{2}$ (Eckstein 1977). The total area explored by the blob of dye, within which the blob folds, is typically (see also Taylor 1953 in a related, but different context), $\Sigma \sim\left(l_{0}+\sqrt{D_{\text {eff }} t}\right) \times\left(s_{0}+\sqrt{D_{\text {eff }} t}\right) \dot{\gamma} t \sim d^{2}(\dot{\gamma} t)^{2}$, growing algebraically in time. Because an exponential will always beat a power law, there will necessary be a time for which the area occupied by the scalar overcomes that visited by the blob (i.e. $\Sigma / \mathcal{A}<1$ ), and from that instant of time, overlaps of the folded scalar filaments will be unavoidable. Such an event is illustrated in figure 16. These overlaps will locally delay the mixing process and therefore affect the whole route of the mixture towards 
homogenization. This aspect, and more generally all aspects regarding the concentration content of the mixture and its evolution, are left for future research.

\section{Conclusions}

Motivated by the need to understand on a firm basis the mixing properties of particulate flows, we have provided a complete characterization of the kinematics of stretchings and consecutive elongations of materials lines in non-Brownian particulate suspensions under a simple macroscopic shear. Our observations rely on high resolution PIV measurements of the interstitial fluid velocity field, and our findings are as follows:

i) Following the Diffusive Strip Method of Meunier 2010, we used the experimentally measured velocity fields to numerically advect passive segments in order to reconstruct the stretching histories of fluid material lines. In agreement with previous theoretical predictions and simulation results, we observe that adding particles in a shear flow changes the very nature of the stretching laws from linear to exponential in strain. The growth rate for the mean elongation are found to closely agree with the largest Lyapunov exponent obtained from 3D numerical simulations (Drazer 2002, Dasan 2002). Besides the mean, our analysis also provides the full statistics of the material lines elongation: the variances of the elongations also grow exponentially in strain and the distributions of elongations converge toward log-normals. This statistics of elongation was characterized for a large range of volume fractions $20 \% \leqslant \phi \leqslant 55 \%$.

ii) Using the same velocity fields, we determined the distribution of the local shear rates intensities and their persistence time. From these, we have shown how the fluid material lines undergo a multiplicative stretching process consisting in a noisy multiplicative sequence of correlated motions. We also discussed the important role of the finite correlation time of the velocity field. The model quantitatively predicts the evolution of the mean and the variance of the elongations of the fluid material lines as well as their evolution towards a log-normal distribution.

iii) We have discussed the importance of this characterization of the flow kinematics to understand how mixing proceeds in sheared particulate suspensions. The exponential stretching results in a mixing time increasing logarithmically with the Péclet number. Moreover, the broad distribution of stretching rates implies a broad distribution of mixing times. The stochastic nature of the stretching process thus allows stretching rates that are smaller than in a pure shear flow. However, our analysis shows that the occurrence of such events becomes negligible at large Péclet number $\left(\gg 10^{5}\right)$ as mixing occurs at larger deformations.

The present study opens the way for a complete description of the mixing process occurring in sheared particulate suspension. In particular, it allows the prediction of the evolution of the concentration distribution $P(C, t)$ (Duplat 2010). A quantitative verification of these predictions requires a specific experimental device that resolves the Batchelor scale $s\left(t_{\text {mix }}\right)$ which corresponds to the transverse dimension of the filaments at the time when diffusion significantly modifies the concentration levels. Such challenging measurements will be addressed in future studies.

We would like to thank P. Meunier for letting us use his DSM code, S. Dagois Bohy, S. Gallier, E. DeGiuli, M. Wyart, O. Pouliquen for having thoughful discussions and P. Cervetti, S. Noel and S. Martinez for helping us build the experimental set-up. This work was supported by ANR JCJC SIMI 9 and by the Labex MEC ANR-10-LABX-0092 et ANR-11-IDEX-0001-02. 


\section{REFERENCES}

Afonso, M. M. And Vincenzi, D. 2005 Nonlinear elastic polymers in random flow J. Fluid Mech. 540, 99-108.

Ahuja A. S. 1975 Augmentation of heat transport in laminar flow of polystyrene suspensions. I. Experiments and results, J. Appl. Phys. 48, 34083416.

Arp P. A. \& Mason S. G. 1976, The Kinetics of Flowing Dispersions IX. Doublets of Rigid Spheres (Experimental), J. Coll. Int. Sci. 61, Issue 4095, 4461.

BAtchelor G. K. 1959 Small-scale variation of convected quantities like temperature in a turbulent fluid. part 1. general discussion and the case of small conductivity, J. Fluid Mech., 5, 113-133.

Boyer F. , Guazzelli E. , And Pouliquen O. 2011 Unifying suspension and granular rheology, Phys. Rev. Let., 107, 188301.

Breedveld V., van den Ende D., Tripathi A. \& Acrivos A. 1998 The measurement of the shear-induced particle and fluid tracer diffusivities in concentrated suspensions by a novel method., J. Fluid Mech., 375, 297318.

Breedveld V., Van den Ende D., Bosscher M., Jongschaap R. J. J. \& Mellema J. 2002 Measurement of the full shear-induced self-diffusion tensor of noncolloidal suspensions, $J$. Chem. Phys. 116, 23.

Buch JR. K.A. \& DAнm W.J.A. 1996 Experimental study of the fine-scale structure of conserved scalar mixing in turbulent shear flows, J. Fluid Mech., 317, Part 1, 2171.

Drazer G., Koplik J., Khusid B. And Acrivos A. 2002 Deterministic and stochastic behaviour of non-Brownian spheres in sheared suspensions, J. Fluid Mech., 460, 307-335.

Chateau X., Ovarlez G., \& Trung K. L. 2008 Homogenization approach to the behavior of suspensions of noncolloidal particles in yield stress fluids, J. Rheol., 52, 489506.

Cocke, W. J. 1969 Turbulent Hydrodynamic Line Stretching: Consequences of Isotropy, Phys. Fluids, 12, (12), 2488-2492.

Cocke, W. J. 1971 Turbulent Hydrodynamic Line-stretching: The Random Walk Limit, Phys. Fluids, 14, (8), 1624-1628.

Culbertson C. T., Jacobson S. C. \& Ramsey J. M. 2002 Diffusion coefficient measurements in microfluidic devices, Talanta, 56, 365373.

Dasan J., Ramamohan T. R., Singh A. And Nott P. 2002 Stress fluctuations in sheared Stokesian suspensions, Phys. Rev. E 66, 021409.

Da Cunha F.R. \& Hinch E. J. 1996 Shear-induced dispersion in a dilute suspension of rough spheres, J. Fluid Mech. 309, 211223.

Dagois-Bohy S., Hormozi S., Guazzelli E., \& Pouliquen O. 2015 Rheology of dense suspensions of non-colloidal spheres in yield-stress fluids, J. Fluid Mech. 776, R2.

Duplat J. \& Villermaux E. 2000 Persistence of Material Element Deformation in Isotropic Flows and Growth Rate of Lines and Surfaces, Eur. Phys. J. B 18, 353-361.

Duplat J. \& Villermaux E. 2008 Mixing by random stirring in confined mixtures, J. Fluid Mech. 617, 51-86.

Duplat J., Innocenti C. \& Villermaux E. 2010 A nonsequential turbulent mixing process, Phys. Fluids 22, 035104.

Eckstein E. C., Bailey D. G. \& Shapiro A. H. 1977 Self-diffusion of particles in shear flow of a suspension, J. Fluid Mech. 79, 191208.

Girimaji, S. S. \& Pope, S. B.. 1990 Material-element Deformation in Isotropic Turbulence J. Fluid Mech. 220, 427-458.

Harlen O. G. AND Koch D. L. 1993 Simple shear flow of a suspension of fibres in a dilute polymer solution at high Deborah number J. Fluid Mech. 252, 187-207.

Le Borgne T., Dentz M. \& Villermaux E. 2015 The lamellar description of mixing in porous media, J. Fluid Mech. 770, 458498.

Lerner E., Dring G., \& Wyart M. 2015 A unified framework for non-Brownian suspension flows and soft amorphous solids, Proc. Natl Acad. Sci USA 109, 47984803.

Lester D. R. , Dentz M., Le Borgne T. 2016 Chaotic Mixing in Three Dimensional Porous Media, arXiv:1602.05270.

Metzger B. \& Butler J. E. 2012 Clouds of particles in a periodic shear flow, Phys. Fluids 24, 021703 . 
Metzger B. \& Butlen J. E. 2012 Irreversibility and chaos: Role of long-range hydrodynamic interactions in sheared suspensions, Phys. Rev. E 82, 051406.

Metzger B., Pham P. \& Buler J. E. 2013 Irreversibility and chaos: Role of lubrication interactions in sheared suspensions, Phys. Rev. E 00, 002300.

Metzger B., Rahli O. \& Yin X. 2013 Heat transfer across sheared suspensions: Role of the shear-induced diffusion, J. Fluid Mech. 724, 527552.

Meunier P. \& Leweke T. 2003 Analysis and minimization of errors due to high gradients in particle image velocimetry. Exp. Fluids 35, 408421.

Meunier P. \& Villermaux E. 2003 How vortices mix, J. Fluid Mech. 476, 213222.

Meunier P. \& Villermaux E. 2010 The diffusive strip method for scalar mixing in two dimensions. J. Fluid Mech. 662, 134-172.

Orszag, S. A. 1970 Comments on 'Turbulent Hydrodynamic Line Stretching: Consequences of Isotropy', Phys. Fluids 13, 2203-2204.

Otтino J.M. 1989 "The Kinematics of Mixing: Stretching, Chaos, and Transport", Cambridge University Press.

RANZ W. E. 1979 Applications of a stretch model diffusion, and reaction in laminar and turbulent flows, AlChE Journal 25, 1.

Shaqfen E. S. G. And Koch D. L. 1992 Polymer stretch in dilute fixed beds of fibres or spheres, J. FLuid Mech. 244, 17-54.

Sierou A. \& Brady J. F. 2004 Shear-induced self-diffusion in non-colloidal suspensions, J. Fluid Mech. 506, 285314.

Sohn C. W. \& Chen M. M. 1984 Heat Transfer enhancement in laminar slurry pipe flows with power law thermal conductivities, J. Heat Trans. 106, 539542.

Souzy M., Yin X., Villermaux E., Abid C. \& Metzger B. 2015 Super-diffusion in sheared suspensions, Phys. Fluids 27, 041705.

TAYloR G. 1953 Dispersion of soluble matter in solvent flowing slowly through a tube, Proc. R. S. London A: 219, 1137.

Trulsson M., DeGiuli E., Wyart M. 2016 Effect of Friction on Dense Suspension Flows of Hard Particles, Arxiv 2016 (https://arxiv.org/abs/1606.07650)..

Wang N. L. \& Keller K. H. 1985 Augmented Transport of Extracellular Solutes in Concentrated Erythrocyte Suspensions in Couette Flow, J. Col. Int. Sc. 103, 210225.

Wang L., Koch D. L., Yin X. \& Cohen C. 2009 Hydrodynamic diffusion and mass transfer across a sheared suspension of neutrally buoyant spheres, Phys. Fluids 21, 033303.

Villermaux, E. \& Duplat, J. 2003 Mixing as an aggregation process Phys. Rev. Letters, 91 (18), 184501-1-4

Villermaux E. 2012 On dissipation in stirred mixtures, Advances in Applied Mechanics, 45, 91-107.

Zydney A. L. \& Colton C. K. 1988 Augmented solute transport in the shear flow of a concentrated suspension, Physico Chem. Hydro. 10, 7796. 